\title{
Transcriptional, epigenetic and metabolic signatures in cardiometabolic syndrome defined by extreme phenotypes.
}

Denis Seyres ${ }^{* 1,2,3}$, Alessandra Cabassi ${ }^{4}$, John J Lambourne ${ }^{1,2,3}$, Frances Burden ${ }^{1,2,3}$, Samantha Farrow $^{1,2,3}$, Harriet McKinney ${ }^{2}$, Joana Batista ${ }^{2}$, Carly Kempster ${ }^{2}$, Maik Pietzner ${ }^{5}$, Oliver Slingsby ${ }^{6,7}$, Thong Huy Cao ${ }^{6,7}$, Paulene A Quinn ${ }^{6,7}$, Luca Stefanucci ${ }^{2,3,8}$, Matthew C Sims ${ }^{2,3,9}$, Karola Rehnstrom ${ }^{2}$, Claire L Adams ${ }^{10}$, Amy Frary ${ }^{2}$, Bekir Ergüener ${ }^{11}$, Roman Kreuzhuber ${ }^{2,12}$, Gabriele Mocciaro ${ }^{13}$, Simona D’Amore ${ }^{14,15,16}$, Albert Koulman ${ }^{5,17,18,19}$, Luigi Grassi ${ }^{1,2,3}$, Julian L Griffin ${ }^{13}$, Leong Loke $\mathrm{Ng}^{6,7}$, Adrian Park $^{14}$, David B Savage ${ }^{10}$, Claudia Langenberg ${ }^{5}$, Christoph Bock ${ }^{11,20,21}$, Kate Downes ${ }^{1,2,22}$, Nicholas J Wareham $^{5}$, Michael Allison ${ }^{14}$, Michele Vacca ${ }^{10,13}$, Paul DW Kirk ${ }^{4,23}$, Mattia Frontini*1,3,8

1. National Institute for Health Research BioResource, Cambridge University Hospitals, Cambridge Biomedical Campus, Cambridge, United Kingdom

2. Department of Haematology, University of Cambridge, Cambridge, Cambridge Biomedical Campus, United Kingdom

3. NHS Blood and Transplant, Cambridge Biomedical Campus, Cambridge, United Kingdom

4. MRC Biostatistics Unit, University of Cambridge, Cambridge, Cambridge Biomedical Campus, United Kingdom

5. MRC Epidemiology Unit, University of Cambridge, Cambridge, UK.

6. Department of Cardiovascular Sciences, University of Leicester, Glenfield Hospital, Leicester, United Kingdom

7. National Institute for Health Research Leicester Biomedical Research Centre, Glenfield Hospital, Leicester, United Kingdom

8. British Heart Foundation Centre of Excellence, Cambridge Biomedical Campus, United Kingdom

9. Oxford Haemophilia and Thrombosis Centre, Oxford University Hospitals NHS Foundation Trust, NIHR Oxford Biomedical Research Centre, Oxford, UK

10. Metabolic Research Laboratories, Wellcome Trust-Medical Research Council Institute of Metabolic Science, University of Cambridge, Cambridge CB2 0QQ, UK

11. CeMM Research Center for Molecular Medicine of the Austrian Academy of Sciences, Vienna, Austria

12. European Molecular Biology Laboratory, European Bioinformatics Institute, Hinxton, UK

13. Department of Biochemistry and the Cambridge Systems Biology Centre, University of Cambridge, The Sanger Building, 80 Tennis Court Road, Cambridge, CB2 1GA, UK.

14. Addenbrooke's Hospital, NIHR Cambridge Biomedical Research Centre, Cambridge University Hospitals NHS Foundation Trust, Cambridge, United Kingdom 
15. Department of Medicine, Aldo Moro University of Bari, Piazza Giulio Cesare 11, 70124 Bari, Italy.

16. National Cancer Research Center, IRCCS Istituto Tumori 'Giovanni Paolo II', Viale Orazio Flacco, 65, 70124 Bari, Italy.

17. MRC Elsie Widdowson Laboratory, Cambridge, United Kingdom.

18. National Institute for Health Research Biomedical Research Centres Core Nutritional Biomarker Laboratory, University of Cambridge, Addenbrooke's Hospital, Cambridge, United Kingdom.

19. National Institute for Health Research Biomedical Research Centres Core Metabolomics and Lipidomics Laboratory, University of Cambridge, Addenbrooke's Hospital, Cambridge, United Kingdom.

20. Ludwig Boltzmann Institute for Rare and Undiagnosed Diseases, Vienna, Austria

21. Department of Laboratory Medicine, Medical University of Vienna, Vienna, Austria

22. East Midlands and East of England Genomic Laboratory Hub, Cambridge University Hospitals NHS Foundation Trust, Cambridge, UK

23. Cambridge Institute of Therapeutic Immunology \& Infectious Disease (CITIID), Jeffrey Cheah Biomedical Centre, Cambridge Biomedical Campus, University of Cambridge, Puddicombe Way, Cambridge CB2 0AW, UK

*To whom correspondence should be addressed. E-mail: Denis Seyres: ds777@medschl.cam.ac.uk, Paul DW Kirk: paul.kirk@mrc-bsu.cam.ac.uk, Mattia Frontini: mf471@cam.ac.uk,m.frontini@exeter.ac.uk

Further information and requests for resources should be directed to and will be fulfilled by the Lead Contact, Mattia Frontini(m.frontini@exeter.ac.uk).

\section{Keywords}

Epigenetics, Metabolites, Lipids, Multi-omics, Obesity, Lipodystrophy, Bariatric Surgery, Classification, Innate Immune Cells

\section{Abstract}

Improving the understanding of cardiometabolic syndrome pathophysiology and its relationship with thrombosis are ongoing healthcare challenges. Using plasma biomarkers analysis coupled with the transcriptional and epigenetic characterisation of cell types 
involved in thrombosis, obtained from two extreme phenotype groups (obese and lipodystrophy) and comparing these to lean individuals and blood donors, the present study identifies the molecular mechanisms at play, highlighting patterns of abnormal activation in innate immune phagocytic cells and shows that extreme phenotype groups could be distinguished from lean individuals, and from each other, across all data layers. The characterisation of the same obese group, six months after bariatric surgery shows the loss of the patterns of abnormal activation of innate immune cells previously observed. However, rather than reverting to the gene expression landscape of lean individuals, this occurs via the establishment of novel gene expression landscapes. Netosis and its control mechanisms emerge amongst the pathways that show an improvement after surgical intervention. Taken together, by integrating across data layers, the observed molecular and metabolic differences form a disease signature that is able to discriminate, amongst the blood donors, those individuals with a higher likelihood of having cardiometabolic syndrome, even when not presenting with the classic features.

\section{Introduction}

Cardiovascular disease (CVD) is the primary cause of death worldwide (17.9 million deaths in $2016,31 \%$ of all deaths $)^{1}$ accompanied by an ever increasing number of overweight and obese individuals, which place a burden of hundreds of billions of dollars on healthcare systems each year ${ }^{2,3}$. Cardiometabolic syndrome (CMS) increases both CVD and type 2 diabetes (T2D) risk ${ }^{4}$. CMS is a cluster of interrelated features including: obesity, dyslipidemia, hyperglycemia, hypertension and non-alcoholic fatty liver disease ${ }^{5}$. These features have overlapping components, including visceral fat deposition, high triglycerides, high low-density lipoprotein (LDL)-cholesterol, high fasting blood glucose, hypertension, decreased high-density lipoprotein (HDL)-cholesterol and low-grade chronic inflammation ${ }^{6-8}$. The therapeutic approaches aim to mitigate these features and include: weight loss strategies ${ }^{9}$, lipid lowering drugs ${ }^{10}$, antiplatelet therapies ${ }^{11}$, glucose lowering ${ }^{12,13}$ and anti-inflammatory drugs $^{14}$. The relationship between cardiometabolic health and body weight is complex ${ }^{15}$. CVD risk varies among individuals of similar body mass index (BMI) depending on adipose tissue (AT) distribution and functionality ${ }^{16-20}$. AT acts as an active endocrine organ ${ }^{21,22}$ and when dysfunctional, plays a major role in metabolic disorders inducing peripheral insulin resistance, and contributing to low-grade chronic inflammation ${ }^{23}$.

Whilst the participation of platelets and neutrophils in thrombosis and that of macrophages in atherosclerotic plaque formation are well established ${ }^{24-26}$, the role of these cell types in 
atherogenesis and CVD onset has been appreciated only recently ${ }^{27}$. Additionally, prolonged exposure to low-grade inflammation is known to modify the functional phenotype of monocytes (an effect named trained immunity ${ }^{28}$ ), platelets ${ }^{29,30}$ and neutrophils ${ }^{31,32}$. The molecular characterisation of these phenotypic changes remains incomplete, motivating the need for extended molecular phenotyping of these cells performed here. Previous multi omics studies in blood cells have identified pathways involved in CVD and obesity, and confirmed whole blood as a source of surrogate biomarkers able to delineate the metabolic status ${ }^{33}$. Several risk score algorithms have been developed to predict the risk of complications associated with obesity ${ }^{34-39}$. However, a number of questions still remain open. CVD may also occur in the absence of other comorbidities and certain events have a better clinical outcome in overweight and obese patients compared with their leaner counterparts (the so-called "obesity paradox") $)^{40}$.

Here, we present the molecular characterization of the transcriptional (RNA sequencing, RNA-Seq) and epigenetic (histone 3 lysine 27 acetylation, H3K27ac; reduced representation bisulfite sequencing, RRBS, and Illumina HumanMethylation450 BeadChip) changes in neutrophils, monocytes, macrophages and platelets in morbidly obese (BMI $>40 \mathrm{~kg} / \mathrm{m} 2$; no obvious genetic cause ${ }^{41}$ ) and in familiar partial lipodystrophy type 2 (hereafter lipodystrophy; causal mutations in PPARG or LMNA genes, as verified by whole genome sequence ${ }^{41}$ ) individuals. We also investigated the reversibility of these molecular changes in the obese group after bariatric surgery. We found that proinflammatory gene expression programs were downregulated, alongside more modest differences in regulatory elements usage and almost no differences in DNA methylation profiles. Altogether, the data indicate a reduced ability of these cells to be activated and undergo extracellular traps (netosis), which was further confirmed by neutrophil and platelet cell functional assays, which showed a reduced ability to adhere, the key initial step during their activation. Lastly, we identified the molecular signatures for CMS and devised a penalised logistic regression approach to stratify individuals in the general population based on their CMS risk.

\section{Results}

\section{Metabolic signatures in the obese and lipodystrophy groups.}

Participants were recruited as follow: controls $(\mathrm{N}=20$; from which metabolically healthy individuals, hereafter lean, were selected, see METHODS), lipodystrophy ( $\mathrm{N}=10)$, morbidly obese referred for bariatric surgery $(\mathrm{N}=11)$ and blood donors (hereafter $\mathrm{BD} ; \mathrm{N}=202)^{42}$. We 
collected age and body weight (BW), and performed plasma biochemistry assays for the following: leptin, adiponectin, insulin, free fatty acid (FFA), glucose (GLC), serum lipid (triglycerides (TG), total cholesterol (TC), high density lipoprotein (HDL-C), low-density lipoprotein (LDL-C)), activity of alanine and aspartate amino-transferases (ALT and AST, respectively) and high-sensitivity C-reactive Protein (hsCRP). Additionally, we computed the following: leptin-adiponectin ratio (LAR), Homeostatic Model Assessment for Insulin Resistance (HOMA-IR) and Adipose Tissue Insulin Resistance (AT-IR) indices (Table 1 and Table S1).First, we wanted to determine if the different groups could be separated based on their plasma biochemistry and anthropometric characteristics. Compared to the other groups, the lipodystrophy group had elevated GLC, TC, TG, ALT, AST, insulin (and consequently HOMA-IR and AT-IR); whereas, HDL-C and LDL-C were decreased. Instead, the obese group, compared to the other 3, had elevated LAR, LDL-C and hsCRP. To visualize how these parameters separate obese, lipodystrophy and $\mathrm{BD}$, we performed a principal component analysis (PCA), which showed that obese, lipodystrophy and BD groups were distributed over distinct, albeit partially overlapping, dimensions (Fig.1A). The first two components (PC1 and PC2) were sufficient to distinguish the different groups (Obese versus Lipodystrophy: $p$ value $=0.002$; Obese versus BD: $p$ value $<2.2 \mathrm{e}-16$; Lipodystrophy versus BD: $p$ value $<2.2 \mathrm{e}-16$; Hotelling's T-squared test with $\mathrm{F}$ distribution). Lipodystrophy and obese were separated from BD along PC1, whilst they were separated from each other along PC2.Loading and contribution analysis (Fig.1B) showed that the main contributors to the separation along PC1 were BW, LAR, hsCRP, AST, ALT, GLC, AT-IR, HOMA-IR and TG. Additionally, BW, LAR, hsCRP separated the obese from the lipodystrophy groups in one direction along PC2, whilst AST, ALT, GLC, AT-IR, HOMA-IR and TG separated them in the opposite direction. The differences observed between the obese and the lipodystrophy groups in plasma biochemistry suggest that, while AT dysfunction is a shared feature, its influences were different in the two groups.

We further characterised the differences between the obese and lipodystrophy groups by investigating plasma metabolites, whose levels are known to be influenced by both extreme phenotypes ${ }^{43,44,45,46}$. We identified and quantified 988 plasma metabolite species (using Metabolon $^{\circledR}$ (METHODS)) and we performed a weighted gene co-expression network consensus analysis (WGCNA) ${ }^{47}$ to create groups of metabolites whose levels were correlated across samples. To identify shared features and to reach the sample size as recommended for such analysis ${ }^{47}$ the obese and lipodystrophy group were analyzed together. This analysis identified 16 clusters of metabolites (named modules, M1 to M16; Table S2). 


\begin{tabular}{|c|c|c|c|c|c|}
\hline & $\begin{array}{l}\text { Blood donors } \\
\qquad(\mathrm{n}=202)\end{array}$ & $\begin{array}{l}\text { Controls } \\
(\mathrm{n}=20)\end{array}$ & $\begin{array}{l}\text { Lipodystrophy } \\
\qquad(\mathrm{n}=10)\end{array}$ & $\begin{array}{l}\text { Obese } \\
(n=11)\end{array}$ & $\begin{array}{l}\text { Post surgery } \\
\qquad(\mathrm{n}=10)\end{array}$ \\
\hline $\begin{array}{l}\text { Adiponectin } \\
\qquad(\mu \mathrm{g} / \mathrm{ml})\end{array}$ & $10.1 \pm 6.3$ & $10.7 \pm 3.7$ & $3.2 \pm 2.3$ & $5.9 \pm 1.9$ & $6.4 \pm 2.6$ \\
\hline AGE(years) & $57.3 \pm 11.1$ & $40.7 \pm 11$ & $45.1 \pm 9.6$ & $46.3 \pm 12.3$ & $43 \pm 12.6$ \\
\hline ALT (U/L) & $34.6 \pm 12$ & $27.1 \pm 7.5$ & $56 \pm 12.7$ & $35.7 \pm 9.4$ & $36.1 \pm 17$ \\
\hline AST (U/L) & $25.5 \pm 11.1$ & $21.8 \pm 6.9$ & $39 \pm 16.8$ & $22.6 \pm 3.8$ & $18.9 \pm 6.9$ \\
\hline AT-IR & $2.6 \pm 2.5$ & $1.9 \pm 2.5$ & $8.4 \pm 7$ & $7.2 \pm 11.2$ & $4.8 \pm 5.4$ \\
\hline BMI $\left(\mathrm{kg} / \mathrm{m}^{2}\right)$ & $26.4 \pm 4.9$ & - & - & $45 \pm 5.1$ & - \\
\hline BW (kg) & $76 \pm 14.9$ & - & $73.2 \pm 9.7$ & $137.9 \pm 35.2$ & - \\
\hline $\mathrm{FFA}(\mu \mathrm{mol} / \mathrm{L})$ & $189.3 \pm 132.8$ & $156.5 \pm 103.3$ & $259.6 \pm 174.9$ & $293.1 \pm 164.6$ & $232.2 \pm 141.5$ \\
\hline GLC (mmol/L) & $5.4 \pm 1.8$ & $4.9 \pm 1$ & $8.3 \pm 3.4$ & $5.3 \pm 0.6$ & $5.3 \pm 1.5$ \\
\hline HDL-C (mmol/L) & $1.6 \pm 0.5$ & $1.7 \pm 0.4$ & $0.8 \pm 0.6$ & $1.3 \pm 0.2$ & $1.3 \pm 0.2$ \\
\hline HOMA-IR & $4.3 \pm 4.9$ & $2.5 \pm 2.2$ & $13 \pm 10.5$ & $7.1 \pm 11.3$ & $8.6 \pm 18.4$ \\
\hline hsCRP (mg/L) & $1.9 \pm 1.8$ & $2.2 \pm 1.2$ & $2.3 \pm 3.3$ & $7.4 \pm 6.9$ & $2.9 \pm 5.6$ \\
\hline Insulin $(\mathrm{pmol} / \mathrm{L})$ & $118.4 \pm 117.1$ & $76.4 \pm 55.6$ & $261.7 \pm 262.3$ & $190.6 \pm 276.2$ & $178.7 \pm 294.3$ \\
\hline LAR & $1.8 \pm 2.1$ & $2 \pm 2.1$ & $2.3 \pm 1.9$ & $13.7 \pm 6.6$ & $5.5 \pm 4.5$ \\
\hline LDL-C (mmol/L) & $2.9 \pm 0.9$ & $2.7 \pm 0.8$ & $1.7 \pm 0.5$ & $2.4 \pm 0.8$ & $2.6 \pm 1$ \\
\hline Leptin (ng/ml) & $14.2 \pm 14.7$ & $19.8 \pm 17.1$ & $7.6 \pm 7.8$ & $74.1 \pm 30.4$ & $29.9 \pm 21$ \\
\hline $\mathrm{TC}(\mathrm{mmol} / \mathrm{L})$ & $5.3 \pm 1.1$ & $4.9 \pm 1$ & $4.2 \pm 1$ & $4.5 \pm 0.8$ & $4.1 \pm 1.4$ \\
\hline $\mathrm{TG}(\mathrm{mmol} / \mathrm{L})$ & $1.6 \pm 0.9$ & $1.2 \pm 0.9$ & $5.6 \pm 5.5$ & $1.9 \pm 0.7$ & $1 \pm 0.4$ \\
\hline
\end{tabular}

Table 1. Descriptive characteristics of the study groups. Average value and standard deviation are reported. 
To determine the relationship between modules, anthropometric traits and plasma biochemistry, we investigated if any correlation existed. Of the 208 tested associations, we found that 11 modules showed significant associations with BW, LAR, TG, HDL-C, LDL-C, ALT and AST in the extreme phenotype groups (FDR adjusted Fisher p values < 0.05; Fig.1C); while no associations were found in the BD cohort (not shown). To determine which modules were associated with each of the two extreme phenotype groups, we analysed the modules eigen-metabolite adjacencies (Fig.1D). The modules formed different clusters, C1 and $\mathrm{C} 2$ were found using extreme phenotype groups, C3 and C4 using BD samples (Fig.S1A). Plotting the average eigen-metabolite value for each cluster (Fig.1E) we showed that $\mathrm{C} 1$ and $\mathrm{C} 2$ represented the obese and lipodystrophy groups respectively, whereas clusters C3 and C4 could not discriminate between obese and lipodystrophy (Fig.S1B). C1 metabolites were significantly enriched in alanine, aspartate and glutamate metabolism, phenylalanine metabolism, nitrogen metabolism and TCA cycle; whereas C2 metabolites we found glycine, serine and threonine metabolism and cysteine and methionine metabolism pathways (Table S2).

Our analysis demonstrated that the two extreme phenotype groups could be identified by their metabolic signatures, associated with clinical parameters, which also set them apart from the general population represented by BD.

\section{Extreme phenotypes influence innate immune cell types and platelets transcriptional and epigenetic signatures.}

Next, we determined the influence of the changes in plasma on neutrophils, monocytes, macrophages and platelets, as these are some of the key players in atherogenesis and thrombus formation ${ }^{48}$ (Fig.2A). We compared gene expression (RNA-sequencing), active chromatin (histone 3 lysine 27 acetylation distribution) and DNA methylation (reduced representation bisulfite sequencing and Illumina arrays) in controls, BD and extreme phenotype groups. For each assays we performed the following comparisons: lean versus obese, lean versus lipodystrophy and obese versus lipodystrophy (Fig.2A and Table S7). For each comparison we identified differentially expressed genes (DEG; Table S8-S11), differentially acetylated regions (DAcR; Table S12-S14) and differentially methylated CpG islands (Table S15-S17) at a FDR 5\%.

Overall, we observed modest changes at transcriptional and epigenetic level in all comparisons (Fig.2B, Fig.2C and Fig.S2B), relatively to those observed in other tissues ${ }^{49,50}$. The largest number of changes was found in active chromatin $(3,616$ DAcR) in the 
comparison between macrophages of the obese and lipodystrophy groups (Fig.S2B) and these were not accompanied by nearly as many changes in gene expression. This indicates that either similar transcriptional outputs were achieved using different regulatory landscapes ${ }^{51}$ or that these cells have been differently primed to respond to acute stimuli. These findings were in agreement with the absence of overlaps between DEG and genes previously associated with trained immunity ${ }^{52}$ in the lean versus obese and lean versus lipodystrophy comparisons. Functional annotation by gene ontology (GO) terms enrichment analysis for the DEG between the lean and obese groups (Fig.2D) found an enrichment for GO terms related to interferon alpha/beta signalling pathway, as well as focal adhesion in DEG up-regulated in macrophages (Table S18). In monocytes, up-regulated DEG were enriched for GO terms related to inflammatory response and down-regulated DEG were enriched in GO terms related to programmed cell death and ion homeostasis (Table S19). In neutrophils, down-regulated DEG were enriched for genes responding to antithrombotic drugs (Table S20). In the comparison between the lean and lipodystrophy groups (Fig.2D), macrophages up-regulated DEG were enriched GO terms related to cholesterol biosynthesis and immune response activation. In monocytes and neutrophils, up-regulated DEG were enriched in terms related to interferon and immune responses. However modest, these changes illustrated how the exposures, to which the cell types involved in the development of atherosclerosis and in thrombus formation are subjected, modify the molecular phenotypes. Similar results have previously been reported for whole blood cell DNA methylation ${ }^{53}$. With the above exception in macrophages, we found that the two extreme phenotype groups were, as expected, more similar to each other than to the lean group overall, again reflecting the underlying AT dysfunction. To determine if these transcriptomic and epigenetic changes are reversible after exposures removal, a second blood sample was taken from the same obese individuals six months after bariatric surgery, and the same assays were performed.

\section{Effect of bariatric surgery on transcriptional and epigenetic landscapes, and cell functions.}

Bariatric surgery is effective in the management of extreme obesity and associated comorbidities, including CMS risk ${ }^{54}$, with well-established long-term benefits on weight loss, diabetes, hypertension and dyslipidemia ${ }^{55}$. While the effect of this intervention has already been reported ${ }^{56,57}$, little is known about the underlying molecular mechanisms. Because we sampled the same individuals robust pairwise comparisons could be used. In plasma biochemistry we observed a decrease for LAR, TG, hsCRP, AT-IR and AST and an increase 
of HDL-C ( $\mathrm{p}$ values: $7.22 * 10^{-6}, 2.63 * 10^{-9}, 4.98 * 10^{-4}, 2.51 * 10^{-2}, 1.48 * 10^{-3}$ and $1.86^{*} 10^{-3}$ respectively; conditional multiple logistic regression, adjusted for age and sex; Fig.3A; Table S1). Transcriptional and epigenetic paired analyses (Fig.3B) identified DEG in macrophages (599), monocytes (1,931), neutrophils (2,571) and platelets (2,883; Table S8-S11), DAcR in monocytes (229) and neutrophils (788; Table S13-S14) and differentially methylated CpGs in macrophages (201), monocytes (48) and neutrophils (198; Table S15-S17).

DEG GO terms enrichment identified amongst the up-regulated pathways: ribosome formation, metabolism of amino acid and proteins, several immune related pathways and cytoplasm translation and amongst the down-regulated pathways: cholesterol metabolic process (through SREBF and miR33 $3^{58}$ ) and mRNA processing pathways (Table S18-S21). We found genes whose expression was reduced in obese, to revert to the levels observed in the lean group: nine in macrophages (RHPN1, DGKQ, TCTEX1D2, MVD, LDL-R, BCAR1, $A N K R D 33 B, F A S N, C O L 5 A 3$; overlap p value $=3.6^{*} 10^{-8}$, hyper-geometric test), seven in monocytes (EPB41L3, LRRC8B, STARD4, ZNF331, SEMA6B, DSC2, RGPD8; overlap p value $\left.=5^{*} 10^{-6}\right)$, five in neutrophils (NAIP, RP11-1319K7.1, LINC01271, LINC01270, DNAH17; overlap $\mathrm{p}$ value $\left.=1.3 * 10^{-5}\right)$ and ten in platelets (CTC-429P9.4, XXbac-BPG300A18.13, RP11-386G11.10, MT-TG, TVP23C-CDRT4, SHE, MPZL3, CLIP1, $R G P D 1, R P L 23 A P 7$; overlap $\mathrm{p}$ value $=6.5^{*} 10^{-5}$ ). These indicate that lipoprotein metabolism (LDL-R), fatty acid synthesis (FASN) and cholesterol transport (STARD4) are restored after surgery. We also found two genes in macrophages $(S N H G 5, E V I 2 A$; overlap p value $=0.03$ ) and three in monocytes (XXbac-BPG32J3.22, MEIS2, MS4A14; overlap p value $=0.03$ ) that move in the directions. While some genes, after bariatric surgery, reverted to expression level observed in lean individuals, the majority of DEG either did not revert to the values observed in lean individuals or were not differentially expressed in the comparison between the obese and lean groups. This suggests that the reduction in inflammatory signatures observed in these four cell types after bariatric surgery was achieved with the establishment, at least in the time frame investigated, of novel gene expression landscapes. Moreover, the overall small number of changes in DNA methylation observed, together with the short life span of the hematopoietic cells analysed, indicated that the change in exposure had little effect on the hematopoietic stem cell epigenome and that the effects observed in animal models ${ }^{59}$ were either species specific or were diluted and then lost with the turnover of the hematopoietic progenitor pool.

The effects of bariatric surgery at organism level were monitored with plasma proteomics. We quantified 3,098 plasma proteins; 604 of which were found to be differentially abundant 
(DAP; Fig.3C and Table S24) above ordinal Q-value of $1 * 10^{-3}$. Proteins whose levels increased after bariatric surgery $(n=72)$ were enriched in GO terms related to tight junction and WNT, PI3K/AKT, sphingolipid signalling pathways. Proteins whose abundance decreased after surgery $(n=532)$ were enriched in the following GO terms: cell cycle and DNA repair, ribosomal RNA metabolism and cell senescence, phagocytosis and $\mathrm{T}$ cell receptor signalling as well as FGF, IL2, VEGF and insulin signalling pathways (Table S25). Amongst these we also found NLRP3, a critical mediator of inflammation ${ }^{60}$ and several histones, normally released by cells undergoing apoptosis and netosis ${ }^{61}$. No changes in full blood count that could explain these changes were observed. We only noted an increase in mean platelet volume $(\mathrm{p}$ value $=0.03$; paired t-test $)$ and a reduction of the lymphocytes $(\mathrm{p}$ value $=0.03)$ and eosinophils $(\mathrm{p}$ value $=0.03$; Table S1) counts. The plasma proteomic results showed that the changes after bariatric surgery were not limited to immune cells. To determine if any of them could be ascribed to a specific tissue, we determined which genes were tissue specific, using the GTEx project database ${ }^{62}$ (Table S26; METHODS). Tibia, coronary and aortic arteries, heart atrial appendage, heart left ventricle, and blood displayed an enrichment for tissue specific genes amongst DAP (p values: $1.6^{*} 10^{-2}, 8^{*} 10^{-3}, 2^{*} 10^{-2}$, $1.8^{*} 10^{-2}, 1.6^{*} 10^{-2}$ and $5^{*} 10^{-2}$, respectively; hyper-geometric test; Table S26). Of the 13 blood specific genes encoding a DAP, six were also differentially expressed in at least one of the cell types (Fig.3D). These six genes have roles in immune response and leptin resistance ${ }^{63}$, immune pathways ${ }^{64}$, neutrophils recruitment during thrombosis ${ }^{65}$ and macrophage differentiation and inflammatory response ${ }^{66}$. The overall decrease observed indicated that vascular integrity, compromised by obesity ${ }^{67}$, was restored, as also observed by Albrechtsen and colleagues ${ }^{68}$.

Furthermore, monocytes and macrophages data allowed us to explore the effect of bariatric surgery on trained immunity ${ }^{52}$, which has been shown to play a role in atherosclerosis ${ }^{69,70}$. Genes displaying an active promoter (H3K4me3), with or without $\beta$-glucan treatment, significantly overlapped with DEG in the obese versus post surgery comparison ( $p$ value = $4.5^{*} 10^{-2}$ and $\mathrm{p}$ value $=7.7^{*} 10^{-3}$; Table S27). This suggested that bariatric surgery had a positive impact on innate immune cells, and indicated that trained immunity acts downstream of the hematopoietic stem cell pool and its effects were diluted and eventually lost with the renewal of the hematopoietic progenitors pool.

To determine the impact of the changes observed at molecular levels on the functional phenotypes of these cell types, we performed functional tests on neutrophils and platelets. After bariatric surgery, neutrophils showed a reduction in their ability to adhere both when 
unstimulated, as well as, when subjected to a variety of stimuli (DTT, LBP, PAM3, PAF and fMLP), but not when treated with TNFalpha or PMA (Fig. 3E). These results were accompanied by a reduction in the cell surface levels of CD16 and CD32, but not CD66b, CD63, CD62L or CD11b (paired t-test, all result in Table S28). Alongside, we also performed platelet functional tests, which showed a reduction in P-selectin surface exposure upon collagen stimulation, but not upon ADP or thrombin stimulation (Fig.3E). These results were accompanied by a reduction in the cell surface levels of fibrinogen receptor (CD61 and CD41b) and CD36 the thrombospondin receptor that acts as scavenger for oxidized LDL. No changes were observed for CD49b, CD42a, CD42b, CD29 and CD9 (paired t-test, all result in Table S28).

The integration of gene expression and proteomic data showed that some of the changes at transcriptional level were directly involved in the reduction of the proinflammatory environment, but also highlighted a conspicuous involvement of other levels of regulation. Notably, we collected across several data layers evidence to suggest a diminished ability of the cells to use neutrophil extracellular traps (NETosis) $)^{71}$ after bariatric surgery (Fig. 5). NETs are formed by chromatin (DNA and histones), granular antimicrobial proteins and cytoplasmic proteins, are normally found at low levels in the circulation ${ }^{72}$, however in the presence of pathogens or sterile inflammation, such as the increase of reactive oxygen species observed in obese individuals ${ }^{73}$, NETs levels are increased. We observed decrease plasma levels of NLRP3 a critical mediator of the inflammasome ${ }^{60}$, RAC2 a protein directly involved in NETs promotion ${ }^{74}$, MYO1G, a protein promoting immune cells interaction ${ }^{75}$ and several histones, core component of the chromatin released during NETosis ${ }^{75}$ (Fig. 3D). Additionally, the upregulated genes in obese individuals indicated increased activity of neutrophils and monocytes (Table S19-S20).

Lastly, we also observed a decrease in the ability of neutrophils to adhere, alongside changes in their surface proteins levels, such as CD16 and CDE32, also previously associated with NETosis $^{76}$ (Fig.3E; Table S28). In addition, genes associated with DAcR in neutrophils post surgery, showed enrichment in the T-cell receptor signaling pathway, in particular Th17 cell differentiation (Table S22), which suggested a restored ability for neutrophils to activate T-cell through NETosis.

The results of the comparisons between lipodystrophy and post bariatric surgery and post bariatric surgery and lean(Fig.S2) are available in Tables S8 to S17.

Altogether, these findings indicate that many key players in thrombus formation have reduced ability to respond to stimuli after bariatric surgery. Our analyses showed that the extreme 
phenotype groups could be separated using each of the different layers of information. Given the limited number of changes identified using single layer univariate comparisons, we sought to identify multivariable signatures by combining information across layers to better characterise and discriminate between the extreme phenotype and lean groups.

\section{Multi-omic signature classification of extreme phenotypes.}

Multivariable selection approaches have provided an effective means to integrate multiple omics layers and elucidate disease signatures ${ }^{77,78}$. We applied this strategy to integrate all the omics layers of this study. Six lean (METHODS) and six obese individuals, for which we had complete measurements on all layers, in monocytes and in neutrophils, were used as training sets. We applied elastic net penalised logistic regression ${ }^{79}$, ideal with a large number of variables and small sample size ${ }^{80}$, to identify signatures associated with an increased probability of belonging to the obese group and therefore to have some or all features associated with CMS (Fig.4A). The variables selected into each signature defined patterns characterising the groups (Fig.4B; Table S29-31) and the biometric variables were used to construct multivariable logistic regression models. All models, single layer or multi-layer trained, allowed us to rank individuals according to their probability of belonging to the obese group (Fig.4C and Fig.S3A). We quantified the $\log \operatorname{loss}{ }^{81}$ (or cross-entropy loss; Fig.4C and Fig.S3A) and demonstrated that the multi-layer model provided the greatest separation, followed by the models trained on the RNA-seq, then those trained on metabolites and methylation. By training the multi-layer predictive model using lipodystrophy and lean individuals, we found that the obese individuals were often predicted to belong to the "lipodystrophy-like" group, thus validating our approach (Fig.S3B). This showed that, while each participant rank might vary across layers, as per our initial hypothesis, those predicted by the integrative model to belong to the extreme phenotype groups, were those individuals with the higher similarity, across all layers, to the training group. It should be noted that amongst the $20 \mathrm{BD}$ that ranked the closest to the obese in the multi-layers model, 4 were in the lowest quartile for weight and 8 were in the lowest quartile for LDL (Table S33), thus confirming that this approach allow to locate those individuals that do not display the most classic CMS presentation. The minor differences observed when comparing the models trained using the lipodystrophy instead of the obese group likely reflect the higher heterogeneity in the latter, lacking a high penetrance genetic cause. External cohorts with similar data layers will be required for a complete validation and to establish the most cost-effective combination of data layers and predictive power. However, because lipidomic 
data from external cohorts were available, we focused on the validation of the lipidomic signature. We prioritised a reduced set of nine lipid species from the signature to test for univariate association with known CMS risk factors (METHODS, Fig.4D), including eight matched lipid species measured in a subset of 1,507 participants in the Fenland study ${ }^{82}$. After correcting for multiple testing, 61\% (225/368) of associations remained significant. Triacylglycerol 52:2 and 50:1 were positively associated with several risks factors (fasting plasma glucose, fasting insulin level, HOMA-IR, a fatty liver index, HbA1c, leptin, LDL-C, hsCRP, TG, BMI, fat mass, ALT, and ferritin; Table S32) and inversely associated with adiponectin and HDL-C. Phosphatidylcholine (40:7), (38:7), (38:6), (35:2) and O (36:2) were inversely associated with all factors except for adiponectin and HDL-C. Further supporting our findings, phosphatidylcholine (38:6 and 36:2) had previously been identified in obesity studies $^{83}$; and, triacylglycerol (50:1 and 52:2) had previously been linked to NAFLD ${ }^{82}$ and $\mathrm{NASH}^{84}$. To assess the specificity of the results, we repeated the analysis with five lipid species randomly selected (METHODS) from those not included in the signature. Only 21\% of associations were significant (49 out of 230 tests). The same pattern of associations was also found in our study (Fig.4D; Table S32), as well as, in a biopsy-confirmed non-alcoholic steatohepatitis (NASH) cohort comprising 73 individuals ${ }^{84}$ (Fig.S4; Table S32). We showed the diagnostic value of the prioritised lipid species through their association with major cardiometabolic risk factors in the Fenland study and in the present study; as well as, albeit not significantly due to the small sample size, in the NASH cohort.

\section{Conclusion}

Our overall goal was to develop an integrative multi-omic strategy to combine information collected across different -omics layers in order to account for the impact of genetic and environmental differences on each of them. We generated data from extreme metabolic phenotype groups to obtain a signature for CMS and then used this signature to determine the cardiometabolic status of a group of individuals (BD) that due to age, are at increased risk of developing CMS. Substantial annotations in our analysis identified the reduction of inflammation and the reduction of the ability to form extracellular traps as key consequences of bariatric surgery in innate immune cells and platelets. Further investigations of the molecular basis underlying the priming of these innate immune cells will help to understand which features, such as small molecules or metabolites, promote abnormal inflammation and extracellular traps formation, providing possible avenues for future clinical treatments. 


\section{Acknowledgments and funding.}

L.S. is supported as $\mathrm{PhD}$ student by British Heart Foundation Cambridge Centre of Excellence; M.C.S is supported by a MRC Clinical Research Training Fellowships (MR/R002363/1); D.B.S is supported by the Wellcome Trust (WT 107064), the MRC Metabolic Disease Unit (MRC_MC_UU_12012.1), and The National Institute for Health Research (NIHR) Cambridge Biomedical Research Centre and NIHR Rare Disease Translational Research Collaboration; K.D. is supported as a HSST trainee by NHS Health Education England; P.D.W.K is supported by Medical Research Council (MC_UU_00002/13). The Human Research Tissue Bank is supported by the NIHR Cambridge Biomedical Research Centre. M.F. is supported by the British Heart Foundation (FS/18/53/33863). D.S. work has been supported in part by an Isaac Newton fellowship to M.F, L.L.N. is supported by the NIHR Leicester Biomedical Research Centre and the John and Lucille Van Geest Foundation.

\section{Conflict of interest.}

The authors have no CoI to declare.

\section{Author contributions}

Conceptualization, D.S., J.J.L, L.G., L.L.N., M.V., P.D.W.K and M.F.; Methodology, D.S., A.C., T.H.C., L.L.N., P.D.W.K and M.F.; Software, D.S., A.C., B.E. and P.D.W.K; Validation, D.S., M.P, C.L. and P.D.W.K; Formal analysis, D.S., A.C., T.H.C., B.E., L.L.N., K.D. and P.D.W.K; Investigation, D.S., A.C., T.H.C., P.A.Q., L.L.N., A.P., P.D.W.K and M.F.; Data curation, D.S., A.C., C.L.A., L.L.N. and P.D.W.K; Writing - Original Draft, D.S., A.C., T.H.C., L.L.N., P.D.W.K and M.F.; Writing - Review \& Editing, D.S., A.C., M.P, T.H.C., L.S., M.C.S., G.M, S.D., L.L.N., A.P., M.V., P.D.W.K and M.F.; Visualization, D.S., A.C., M.P, P.D.W.K and M.F.; Funding Acquisition, M.F.; Resources, J.J.L, F.B., S.F, H.M, J.B., C.K., A.K., L.L.N., D.B.S., C.L., C.B., K.D., M.A., M.V., N.J.W. and M.F.; Supervision, L.L.N. P.D.W.K. and M.F, Project administration, M.F. 


\section{Methods}

The datasets generated during this study are available at EGA under study ID EGAS00001003780.

The codes generated during this study and all supplementary tables are available at GitLab https://gitlab.com/dseyres/extremephenotype.

\section{Patients recruitment and ethics}

Obese individuals referred for obese surgery by the obesity clinic and lipodystrophy patient cared for by the National Severe Insulin Resistance Service respectively, both based at Addenbrooke's hospital, Cambridge University Hospitals were recruited to this study together with healthy individuals. Informed consent was obtained under the "Inherited Platelet Disorders" ethics (REC approval 10/H0304/66 for patients and 10/H0304/65 for healthy controls, NRES Committee East of England-Cambridge East).

BluePrint work package 10 (WP10) volunteers (representing the blood donors, "BD”, cohort) were recruited amongst NHS Blood and Transplant donors after informed consent under the "A Blueprint of Blood Cells" ethical approval (REC approval 12/EE/0040 NRES Committee East of England-Hertfordshire).

"BioNASH" Cohort consisted of 73 consecutive patients recruited at the NASH Service at the Cambridge University Hospital. All the patients had a clinical diagnosis of NAFLD (patients with alternate diagnoses and etiologies were excluded) and histology scored. This study was approved by the local Ethics Committee; all patients gave their informed consent for the use of data (Biochemistry and clinical history) and samples for research purposes. The principles of the Declaration of Helsinki were followed.

\section{Cell types isolation}

Whole blood $(50 \mathrm{ml})$ in citrate tubes was obtained after informed consent. Platelet rich plasma (PRP) was separated from the cellular fraction by centrifugation $(20$ ', $150 \mathrm{~g}$ and very gentle break) for platelet isolation. Platelets were then isolated from PRP after 2 more spins as above and leukodepleted using anti CD45 Dynabeads (Thermofisher) following the manufacturer's instructions. Purified platelets were stored in TRIzol (Invitrogen) until RNA extraction. The remaining cells were resuspended in buffer 1 and separated on a Percoll gradient. Neutrophils were harvested from the red blood cell pellet after red cell lysis $(4.15 \mathrm{~g}$ $\mathrm{NH} 4 \mathrm{Cl}, 0.5 \mathrm{~g} \mathrm{KHCO} 3$ and $18.5 \mathrm{mg}$ EDTA (triplex III, $0.01 \%$ ) in $500 \mathrm{ml}$ of water) and 
aliquots prepared for RNA extraction (TRIzol), DNA extraction for RRBS (snap frozen pellet) and ChIP-Seq (formaldehyde fixation, see below). Monocytes were isolated from the peripheral blood mononuclear cell (PBMC) layer by CD14 positive selection (Miltenyi) and aliquots prepared for RNA extraction (TRIzol), DNA extraction for RRBS (snap frozen pellet) and ChIP-Seq (formaldehyde fixation, see below). Macrophages were cultured by plating 14*106 PBMC resuspended in $2 \mathrm{ml}$ macrophage media (Macrophage-SFM [with L-Glutamine without Antibiotics], Fisher Scientific UK LTD). After 1h 30' non adherent cells were removed and $1 \mathrm{ml}$ fresh macrophage media added together with $400 \mu 1$ of autologous serum. Culture media was replaced after 3 or 4 days. On day 7 cells were harvested for RNA extraction (TRIzol), DNA extraction for RRBS (snap frozen pellet) and ChIP-Seq (formaldehyde fixation). Cell purity was determined by flow cytometry as follows: neutrophils CD66b (BIRMA17c, FITC, 9453 https://ibgrl.blood.co.uk/), CD16 (VEP13, PE, 130-091-245 Miltenyi) and CD45 (HI30, PE-CY5.5, MHCD4518 Invitrogen); monocytes CD14 (M甲P9, FITC, 345784 BD), CD16 (B73.1 / leu11c, PE, 332779 BD), CD64(10.1, PerCP-Cy5.5, 561194 BD), CD45 (HI30, PE-CY7, MHCD4512 Invitrogen); macrophages panel 1: CCR7/CD197 (150503, FITC 561271 BD), CD25-PE MACS 120-001-311 (10ul/test), CD14 (TuK4, PE-Cy5.5, MHCD1418 Invitrogen), CD40 (5C3, PE-Cy7, 561215 BD). Panel 2: CD206 (19.2, PE, 555954 BD), CD36 (SM $\boldsymbol{\Phi}$, FITC, 9605-02 Southern Biotech), CD45 (HI30, PE-Cy5.5, MHCD4518 Invitrogen). Samples whose purity was below $90 \%$ were discarded. BD samples isolation has been extensively described in Chen et al..

\section{RNA sequencing}

RNA extraction

RNA extraction from samples stored in TRIzol was performed following the manufacturer's instructions. Briefly, tubes were retrieved in small batches and thawed on ice. Prior to extraction samples were vortexed for $30^{\prime \prime}$ to ensure complete lysis and let for $5^{\prime}$ at room temperature. Samples were then transferred to heavy phase lock tubes (5prime) to separate RNA in the aqueous phase from the organic phase. RNA was precipitated from the former with isopropanol and glycogen. The RNA pellet was resuspended in RNase free water. Purified RNA was stored in single use aliquots. Each sample was quality controlled by a Bioanalayser (Agilent) and quantified via Qubit (Thermofisher).

\section{Library preparation and sequencing}

For cell types isolated from obese and lipodystrophy patients and day controls we used 100 ng of total RNA for neutrophils, monocytes and macrophages and $200 \mathrm{ng}$ for platelets. o 
libraries were prepared for sequencing using the Kapa stranded RNA-Seq kit with riboerase (Roche) according to the manufacturer's instructions and sequenced 150bp paired end on Illumina HiSeq 2500 or Illumina HiSeq 4000. BD RNA-Seq data (extensively described in Chen et $\mathrm{al}^{42}$ ) were retrieved from European Genome-phenome Archive (EGA) - EMBL-EBI after application to the Data Access Committee.

\section{Quantification}

FastQ files were first checked for sequencing quality using FastQC (v.0.11.2) [https://www.bioinformatics.babraham.ac.uk/projects/fastqc/] and quality trimmed with TrimGalore! (v.0.3.7) [https://www.bioinformatics.babraham.ac.uk/projects/trim_galore/]. Transcript-level abundance was estimated using Kallisto (v0.42) $)^{85}$ with 100 bootstrap iterations in single-end mode for extreme phenotype samples in order to minimize technical batch effect with BD cohort. Transcript abundances were then summarized to gene-level with Tximport R package (v1.9) ${ }^{86}$ by using tximport function and Ensembl reference transcriptome (Ensembl Genes 96) ${ }^{86,87}$. This step provides an input count matrix for DESeq2 $(\mathrm{v} \cdot 1.21 .21)^{88}$. DESeq2 was used to normalize counts by library size and transformed by variance stabilisation (VST). We corrected for sequencing batch effect by using Combat (from sva R package (v.3.29.1) $)^{88,89}$ and individual status as covariate. Non-autosomal genes and those with no or low variance across individuals were removed. The final gene sets (including coding and non-coding genes) were formed of 10,925 genes for monocytes and of 26,634 for neutrophils. Quality metrics are reported in table $\mathbf{S 4}$.

\section{Differential analysis}

For differential analysis, transcript-level abundance was estimated by Kallisto with 100 bootstrap iterations in paired-end mode for each group (obese, post surgery, lipodystrophy patients and lean individuals) using Ensembl reference transcriptome (Ensembl Genes 96). Transcript abundances were then summarized to gene-level with Tximport R package (v1.9) by using tximport function and DESeq2 object was created using DESeqDataSetFromTximport function from DESeq2 R package (v.1.21.21). Differential analysis was performed using Deseq function from DESeq2 and we used age and gender as covariates. Log fold changes were corrected with lfcShrink function from DESeq2. Genes with FDR $<5 \%$ were marked as differentially expressed. For obese versus post surgery comparison, we considered only paired samples ([S01RS6;S022QS][S01Y9G;S022UK][S01WCI;S0232Z][S01TEQ;S0234V][S01WXD;S02 3EB][S01WFC;S023F9][S01Y7K;S023H5][S022TM;S023PQ][S01XJ0;S023RM][S01SYR; 
S0240Z][S022GB;S0245P]) and therefore performed a paired analysis by adding relationship information as covariate in the design formula.

Functional annotation was performed with genes differentially expressed for each cell-types and comparisons, taking into account fold change direction. Lists of genes were submitted to EnrichR using the R package EnrichR (v.1.0) ${ }^{90,91}$ and the following databases: BioCarta_2016, DSigDB, GO_Biological_Process_2018, GO_Cellular_Component_2018, GO_Molecular_Function_2018,HMDB_Metabolites, KEGG_2019_Human, Reactome_2016 and WikiPathways_2015. To facilitate gene lists submission, we developed an R shiny interface to EnrichR (https://blueprint.haem.cam.ac.uk/EnrichR_Interface/).

\section{Chromatin Immunoprecipitation sequencing}

\section{Sample preparation}

Cells were fixed immediately after purification with $1 \% \mathrm{w} / \mathrm{v}$ formaldehyde for $10 \mathrm{~min}$ and quenched using $125 \mathrm{mM}$ glycine before washing with PBS. Samples were sonicated using a Bioruptor (Diagenode), final SDS concentration of $0.1 \% \mathrm{w} / \mathrm{v}$ for 9 cycles of $30 \mathrm{~s}$ 'on' and 30 s 'off', and immunoprecipitated using an IP-Star Compact Automated System (Diagenode) using the histone H3K27ac antibody C15410196 (lot 1723-0041D) Diagenode. Immunoprecipitated and input DNA were reverse cross-linked (65 $\mathrm{C}$ for $4 \mathrm{~h}$ ), treated with RNase and Proteinase K (65 C for $30 \mathrm{~min})$.

\section{Library preparation and sequencing}

DNA was recovered with Concentrator 5 columns (Zymo) and prepared for sequencing using MicroPlex Library Preparation Kit v2 (C05010012, Diagenode). Libraries analysed using High Sensitivity Bioanalyzer chips (5,067-4,626, Agilent), quantified using qPCR Library Quantification Kit (KK4824, Kapa Biosystems), pooled and sequenced with a 50bp single end protocol on Illumina HiSeq 2500 or Illumina HiSeq 4000.

\section{Peak calling and quantification}

FastQ files were first checked for sequencing quality using FastQC (v.0.11.2) and quality trimming were applied on reads with TrimGalore! (v.0.3.7). Trimmed FASTQ files were aligned to the human genome (Ensembl GRCh38.80) with BWA (v.0.7.12) ${ }^{92}$ aln and samse functions with default parameters. Low mapping quality reads (-q 15), multi-mapped and duplicate reads were marked and removed with respectively samtools (v.1.3.1) ${ }^{93}$ and picard (http://broadinstitute.github.io/picard v.2.0.1).

A combination of quality metrics was used to assess sample quality: number of uniquely mapped reads, number of called peaks, NSC (Normalized strand cross-correlation) and RSC 
(relative strand cross-correlation) computed with Phantompeakqualtools (v.1.2) ${ }^{94,95}$, area under the curve (AUC), X-intercept and Elbow Point computed with plotFingerPrint function from deepTools suite (v.3.0.2) ${ }^{96}$ with --skipZeros --numberOfSamples 50000 options. Peaks were called with MACS2 (v.2.1.1) with --nomodel --shift -100 --extsize 200, a qvalue threshold of 1e-3 options and celltype matching input file scaled to sample read number. We used MACS2 randsample function to downscale inputs. We then computed a score by summing values obtained for each range of these metrics. We applied a threshold of -3 (total) to select the best quality data.

\begin{tabular}{|c|c|c|c|c|c|}
\hline & -2 & -1 & 0 & 1 & 2 \\
\hline $\begin{array}{l}\text { Uniq reads } \quad\left(\begin{array}{ll}\% & \text { raw } \\
\text { reads })\end{array}\right.\end{array}$ & $<20$ & $\begin{array}{l}>=20 \quad \text { and } \\
<40\end{array}$ & $\begin{array}{l}>=40 \quad \text { and } \\
<60\end{array}$ & $\begin{array}{l}>=60 \quad \text { and } \\
<80\end{array}$ & $>=80$ \\
\hline Encode - NSC & $<0.9$ & $\begin{array}{l}>=0.9 \quad \text { and } \\
<1\end{array}$ & $>=1$ and $<1.1$ & $\begin{array}{l}>=1.1 \text { and } \\
<1.2\end{array}$ & $>=1.2$ \\
\hline Encode - RSC & $<0.8$ & $\begin{array}{l}>=0.8 \quad \text { and } \\
<0.9\end{array}$ & $>=0.9$ and $<1$ & $\begin{array}{l}>=1 \quad \text { and } \\
<1.1\end{array}$ & $>=1.1$ \\
\hline Deeptools - AUC & $>=0.4$ & $\begin{array}{l}>=0.3 \quad \text { and } \\
<0.4\end{array}$ & $\begin{array}{l}>=0.2 \quad \text { and } \\
<0.3\end{array}$ & $\begin{array}{l}>=0.1 \quad \text { and } \\
<0.2\end{array}$ & $<0.1$ \\
\hline $\begin{array}{l}\text { Deeptools } \\
\text { X-intercept }\end{array}$ & $>=0.3$ & $\begin{array}{l}>=0.2 \quad \text { and } \\
<0.3\end{array}$ & $\begin{array}{l}>=0.15 \text { and } \\
<0.2\end{array}$ & $\begin{array}{l}>=0.1 \quad \text { and } \\
<0.15\end{array}$ & $<0.1$ \\
\hline $\begin{array}{l}\text { Deeptools - Elbow } \\
\text { point }\end{array}$ & $<0.65$ & $\begin{array}{l}>0.65 \text { and } \\
<0.75\end{array}$ & $\begin{array}{l}>0.75 \quad \text { and } \\
<0.85\end{array}$ & $\begin{array}{l}>0.85 \text { and } \\
<0.95\end{array}$ & $>0.95$ \\
\hline Peak number & $\begin{array}{l}<(\mathrm{e}-100 \\
00)\end{array}$ & $\begin{array}{l}>=(\mathrm{e}-10000) \\
\text { and } \\
<(\mathrm{e}-5000)\end{array}$ & $\begin{array}{l}>=(\mathrm{e}-5000) \\
\text { and } \\
<(\mathrm{e}-2000)\end{array}$ & $\begin{array}{l}>=(\mathrm{e}-2000) \\
\text { and }<\mathrm{e}\end{array}$ & $\begin{array}{l}>=\mathrm{e} \text { and } \\
<(\mathrm{e}+250 \\
00)\end{array}$ \\
\hline
\end{tabular}

To build ChIP-Seq layer for integrative analysis, we defined a master set of peaks and quantified H3K27ac ChIP-Seq signals under these peaks. Peaks shared by at least 5 individuals were merged using R package DiffBind (v2.9) ${ }^{97}$. We obtained 67,763 and 49,188 peaks for monocytes and neutrophils, respectively. Minimum merged peak size was $244 \mathrm{bp}$ and $235 \mathrm{bp}$, median peak size 1,392bp and 1,648b and maximum peak size 75,534bp and $60,528 \mathrm{bp}$ for monocytes and neutrophils, respectively. We didn't filter out very large merged peaks as they represent less than $3 \%$ of total peaks and indicate large acetylated regions. Read counts under merged peaks were TMM normalized using effective library size and logit transformed into count per million (CPM). Sequencing center batch effect was corrected with Combat (from sva R package (v.3.29.1)) using individual status (Patient/Donor) as covariate. 
Non-autosomal and no or low variance peaks across individuals were removed. The final master set of peaks counted 25,595 regions in monocytes and 26,300 regions in neutrophils. Quality metrics are reported in table S3.

\section{Differential analysis}

For differential analysis, we used DiffBind with the built-in DEseq2 method for statistical analysis. We merged peaks present in at least 50\% of individuals and asked that all individuals have a FRiP value (Fraction of Reads in Peaks) over 5\%. We then applied a FDR threshold of 5\% to select $\mathrm{H} 3 \mathrm{~K} 27 \mathrm{ac}$ peaks differentially acetylated peaks. We used age and gender as covariates. For obese versus post surgery comparison, we considered only paired samples and therefore performed a paired analysis by using the block factor in DEseq2. Differentially acetylated regions (DAcR) were annotated with HOMER (v.4.10) $)^{98}$, annotatePeak function and $\mathrm{Hg} 38$ RefSeq genome annotation (http://homer.ucsd.edu/homer/data/genomes/hg38.v6.0.zip).

Functional annotation was performed on genes within a window of $10 \mathrm{~kb}$ around each DAcR, taking into account fold change direction. Similarly to RNA-Seq, lists of genes were submitted to EnrichR interrogating the same databases. Annotation results are available in table S22.

\section{Illumina 450K arrays and reduced representation bisulfite sequencing (RRBS)}

Arrays and libraries preparation and sequencing

BD Infinium Human Methylation 450 arrays (Illumina) were retrieved from the European Genome-phenome Archive (EGA) - EMBL-EBI. DNA extraction and array generation have been described in detail in Chen et al. $^{42}$. Briefly, cells were lysed using guanidine hydrochloride, sodium acetate and protease lysis buffer. DNA was extracted using chloroform and precipitated in ethanol prior to washing and resuspension in ultra-pure water. 500 ng of DNA for each monocyte and neutrophil sample was randomly dispensed onto a 96-well plate to reduce batch effects. Samples were bisulfite-converted using an EZ-96 DNA Methylation MagPrep Kit (Zymo Research) following the manufacturer's instructions with optimized incubation conditions (i.e., 16 cycles of 95C for $30 \mathrm{~s}, 50 \mathrm{C}$ for $60 \mathrm{~min}$; followed by 4C until further processing). Purified bisulfite-treated DNA was eluted in $15 \mathrm{~mL}$ of M-Elution Buffer (Zymo Research). DNA methylation levels were measured using Infinium Human Methylation 450 arrays (Illumina) according to the manufacturer's protocol.

For RRBS, $100 \mathrm{ng}$ of genomic DNA were digested for $6 \mathrm{~h}$ at $65^{\circ} \mathrm{C}$ with $20 \mathrm{U}$ TaqI (New England Biolabs) and $6 \mathrm{~h}$ hours at $37^{\circ} \mathrm{C}$ with $20 \mathrm{U}$ of MspI (New England Biolabs) in $30 \mu \mathrm{l}$ of 
1x NEBuffer 2. To retain even the smallest fragments and to minimize the loss of material, end preparation and adaptor ligation were performed in a single-tube setup. End fill-in and A-tailing were performed by addition of Klenow Fragment 3' --> 5' exo- (Enzymatics) and dNTP mix (10 mM dATP, 1 mM dCTP, 1 mM dGTP New England Biolabs). After ligation to methylated Illumina TruSeq LT v2 adaptors using T4 DNA Ligase rapid (Enzymatics), the libraries were size selected by performing a $0.75 \mathrm{x}$ clean-up with AMPure XP beads (Beckman Coulter). The libraries were pooled based on qPCR data and subjected to bisulfite conversion using the EZ DNA Methylation Direct Kit (Zymo Research) with changes to the manufacturer's protocol: conversion reagent was used at $0.9 \mathrm{x}$ concentration, incubation performed for 20 cycles of $1 \mathrm{~min}$ at $95^{\circ} \mathrm{C}, 10 \mathrm{~min}$ at $60^{\circ} \mathrm{C}$ and the desulphonation time was extended to $30 \mathrm{~min}$. These changes increase the number of $\mathrm{CpG}$ dinucleotides covered, by reducing double-strand break formation in larger library fragments. Bisulfite-converted libraries were enriched KAPA HiFi HS Uracil+ RM (Roche). The minimum number of enrichment cycles was estimated based on a qPCR experiment. After a 1x AMPure XP clean-up, library concentrations were quantified with the Qubit Fluorometric Quantitation system (Life Technologies) and the size distribution was assessed using the Bioanalyzer High Sensitivity DNA Kit (Agilent).

\section{Processing and quantification}

All Infinium Human Methylation 450 array data pre-processing steps were carried out using established analytical methods incorporated in the R package RnBeads (v.1.13.4) ${ }^{99}$. First, we performed background correction and dye-bias normalization using $\mathrm{NOOB}^{100}$, followed by normalization between Infinium probe types with SWAN ${ }^{101}$. Next, we filtered out probes based on the following criteria: median detection $\mathrm{p}$ value 0.01 in one or more samples; bead count of less than three in at least $5 \%$ of samples; ambiguous genomic locations ${ }^{102}$; cross-reactive and SNP-overlapping probes ${ }^{103}$.

The RRBS samples were sequenced on Illumina HiSeq3000 platform in 50bp single-end mode. Base calling was performed by Illumina Real Time Analysis (v2.7.7) software and the base calls were converted to short reads using Illumina2bam (1.17.3 https://github.com/wtsi-npg/illumina2bam) tool before de-multiplexing (BamIndexDecoder) into individual, sample-specific BAM files. Trimmomatic (v0.32) ${ }^{104}$ was used for trimming the adapter sequences. Trimmed short read sequences were aligned onto the GRCh38/hg38 human reference genome with $\operatorname{BSMAP}(\mathrm{v} 2.90)^{105}$ aligner in RRBS mode which was optimized for aligning the RRBS data while being aware of the restriction sites and with the following options: -D C-CGG -D T-CGA -w 100 -v 0.08 -r 1 -p 4 -n 0 -s 12 -S 0 -f 5 -q 0 -u 
-V 2. R package RnBeads was used to filter out low confidence sites: sites overlapping any SNP, having a coverage lower than 5 and high coverage or missing in more than $5 \%$ or individuals were filtered out. Integration analysis required to attenuate technology effect between $450 \mathrm{~K}$ arrays and RRBS. To this goal, we generated RRBS data for 14 BluePrint donors for which we already have $450 \mathrm{~K}$ array data in monocytes, and 9 in neutrophils. We first removed non reproducible sites between technologies as follows: for monocytes and neutrophils, 1) liftover $450 \mathrm{~K}$ sites to $\mathrm{Hg} 38$ using UCSC liftover tool ${ }^{106}$, 2) keep overlapping sites between array and RRBS, 3) filter out sites with high variation in methylation percentage observed in more than $70 \%$ of individuals. We excluded 844 and 1,127 sites for monocytes and neutrophils respectively. We have also excluded sites on sex chromosomes and imputed missing values using $\mathrm{KNN}$ networks (impute.knn function from impute $\mathrm{R}$ package (v.1.55.0)) [Hastie T, Tibshirani R, Narasimhan B, Chu G (2019). impute: impute: Imputation for microarray data.] with 10 nearest neighbors. Finally, we adjusted for batch effects using an empirical Bayesian framework, as implemented in the ComBat function of the R package SVA (v.3.29.1) and individual status as covariate, transformed beta values to $\mathrm{M}$ values using beta2m function in $\mathrm{R}$ package lumi (v.2.33.0 ${ }^{107,108}$, normalize by quantile using normalize.quantiles function from $\mathrm{R}$ package preprocessCore (v.1.43.0) [Bolstad B (2019). preprocessCore: A collection of pre-processing functions.] and remove zero or low variance sites. The final data matrix used for multi-omic integration, comprised DNA methylation M-values across 24,311 CpG sites and 210 samples in monocytes and 24,217 CpG sites and 203 samples in neutrophils.Quality metrics are reported in table S5 and S6.

\section{Differential analysis}

For differential analysis, we used the methylKit R package (v.1.8.1) ${ }^{109}$ and we compared only RRBS data. We first extracted methylation ratios from BSMAP mapping results with methratio.py python script provided with BSMAP. We then removed all sex chromosomes sites and filtered out non-retained sites from RnBeads RRBS processing. Finally, we used the methRead function from methylKit $\mathrm{R}$ package in CpGs context at base resolution to read in the input files and calculateDiffMeth function correcting for overdispersion (overdispersion="MN") and applying Chisq-test. We used age and gender as covariates. Q Values are then computed using the SLIM method ${ }^{109,110}$. We applied two thresholds: difference of methylation $>25$ and qualue $<0.05$ and retrieved differentially methylated sites (DMS) with getMethylDiff function specifying type="hypo" or type="hyper" option to get down and up methylated CpGs respectively. 
For obese (pre) versus post surgery comparison, we considered only paired samples and therefore performed a paired analysis. DMS were annotated with HOMER (v.4.10), annotatePeak function and $\mathrm{Hg} 38$ RefSeq genome annotation (http://homer.ucsd.edu/homer/data/genomes/hg38.v6.0.zip).

Functional annotation was performed on genes within a window of 10kb around each DMS, taking into account fold change direction. Similarly to RNA-Seq and ChIP-Seq, lists of genes were submitted to EnrichR interrogating the same databases. Annotation results are available in table S23.

\section{Plasma biochemistry assays}

Plasma biochemistry assays were performed in the Core Biochemical Assay Laboratory, Cambridge University Hospitals (https://www.cuh.nhs.uk/core-biochemical-assay-laboratory) as described in supplementary material and methods. Homeostatic Model Assessment for Insulin Resistance (HOMA) score as follows: (glucose (mg/dL) x insulin (mIU/L)) / 405, and adipose tissue insulin resistance (AT) score as follows: insulin $(\mu \mathrm{U} / \mathrm{mL}) \times$ free fatty acids $(\mathrm{mmol} / \mathrm{L})$.

\section{Plasma metabolites measurement}

\section{Metabolites quantification}

Metabolites profiling of obese and lipodhystrophy patients, day controls and blood donors (BD participants) was performed by Metabolon Inc. (https://www.metabolon.com/) using their standard protocol. Briefly, Metabolon analytical platform incorporates two separate ultra-high performance liquid chromatography/tandem mass spectrometry (UHPLC/MS/MS2) injections and one gas chromatography GC/MS injection per sample. The UHPLC injections are optimized for basic species and acidic species. The numbers of compounds of known structural identity (named biochemicals) as well as compounds of unknown structural identity (unnamed biochemicals) detected by this integrated platform were respectively of 793 and 362 for the first batch and 947 and 433 for the second batch (with an overlap of 786 and 359 compounds respectively). All samples were rescaled to set the median to 1 , missing values were imputed using KNN networks (impute.knn function from impute R package (v.1.55.0) with the following options: number of nearest neighbors $=10$, maximum missing values per metabolites $<50 \%$ and maximum missing values for individuals $<80 \%$.) Finally, we adjusted for batch effects using the ComBat function of the R package SVA (v.3.29.1) and individual status as covariate. 


\section{Plasma lipids measurement}

Plasma was frozen in dry ice immediately after collection and stored at -80C until analysis. Samples were prepared essentially as previously described ${ }^{111}$. Briefly, a $15 \mu \mathrm{L}$ sample, controls and blanks were placed in a predefined random order across 96-well plates (Plate+, Esslab, Hadleigh, UK). To which, $750 \mu \mathrm{L}$ methyl tert-butyl ether was added, along with 150 $\mu 1$ of internal standard mix, containing the following six internal standards (IS): 1,2-di-o-octadecyl-sn-glycero-3-phosphocholine $(0.6$ $\mu \mathrm{M})$ 1,2-di-O-phytanyl-sn-glycero-3-phosphoethanolamine (1.2 $\mu \mathrm{M})$, C8-ceramide $(0.6 \mu \mathrm{M})$, N-heptadecanoyl-D-erythro-sphingosylphosphorylcholine $(0.6 \mu \mathrm{M})$, undecanoic acid $(0.6 \mu \mathrm{M})$, and trilaurin $(0.6 \mu \mathrm{M})$, (Avanti Polar Lipids and SIgma Aldrich). Quality controls were derived from pooling all samples and serially diluting with chloroform. $25 \mu 1$ of the sample/IS mixture was transferred to a glass coated 384 well plate and $90 \mu \mathrm{l}$ mass spectrometry (MS) mix [7.5mM NH4Ac IPA:MeOH (2:1)] added and then sealed. Lipidomics was performed using chip-based nanospray with an Advion TriVersa Nanomate (Advion) interfaced to the Thermo Exactive Orbitrap (Thermo Scientific). Briefy, a mass acquisition window from 200 to $2000 \mathrm{~m} / \mathrm{z}$ and acquisition in positive and negative modes were used with a voltage of $1.2 \mathrm{kV}$ in positive mode and $-1.5 \mathrm{kV}$ in negative mode and an acquisition time of $72 \mathrm{~s}$. Raw spectral data were processed as previously described ${ }^{112}$. Raw data were then converted to .mzXML (usingMSconvert ${ }^{113}$ with peakpick level 1), parsed with $\mathrm{R}$ and 50 spectra per sample (scan from 20 to 70 ) were averaged using XCMS42, with a signal cutoff at 2000. Te fles were aligned using the XCMS ${ }^{114,115}$ grouping function using "mzClust" with a m/z-window of $22 \mathrm{ppm}$ and a minimum coverage of $60 \%$. Compound annotation was automated using both an exact mass search in compound libraries as well as applying the referenced Kendrick mass defect approach. Signal normalisation was performed by summing the intensities of all detected metabolites to a fixed value to produce a correction factor for the efficiency of ionisation. Exact masses were fitted to the lipid species library and subsequently annotated to the peak as described before ${ }^{84}$.

\section{Plasma proteomics}

Sample preparation

Plasma was precleared by centrifugation at 3,000 g for 10 minutes and bound to $100 \mu \mathrm{L}$ of calcium silicate matrix (CSM, $4 \mathrm{mg} / \mathrm{mL}$ ) by rotation for 1 hour. The sample was centrifuged at $14,000 \mathrm{~g}$ for 1 minute and the supernatant was removed for further analysis. The pellet was washed in ammonium bicarbonate $(50 \mathrm{mMoL}, 1 \mathrm{~mL}) 3$ times using the same centrifugation 
settings. The sample was then reduced for 30 minutes at $65^{\circ} \mathrm{C}$ using $200 \mu \mathrm{L}$ of DL-dithiothreitol (DTT) premix (ADC 2\%: ammonium bicarbonate $50 \mathrm{mMoL}$ : DTT $1 \mathrm{MoL}$ in the ratio of 50:49:1) and alkylated for 30 minutes in the dark with iodoacetamide (IAA) at $20 \mathrm{mMoL}$. Ammonium bicarbonate was added to dilute the ADC to $0.5 \%$. Trypsin was added in the ratio of $1: 25$ trypsin to plasma and incubated overnight at $37^{\circ} \mathrm{C}$. The $\mathrm{ADC}$ was precipitated with $1 \%$ formic acid (FA) and centrifuged at $14,000 \mathrm{~g}$ for 10 minutes. The peptides were isolated using solid phase EMPORE C18 discs which had been washed with 1 stem of methanol and 3 stem of $0.1 \%$ FA. The sample was left to bind to the column for 30 minutes before washing with $0.1 \%$ FA and eluting with $60 \%$ acetonitrile (ACN) with $0.1 \%$ FA and then $80 \%$ ACN with $0.1 \%$ FA. The ACN was removed by speed vacuum for 1 hour 15 minutes and freeze dried overnight. Peptide suspended in $30 \mu \mathrm{L}$ of $0.1 \%$ FA and a peptide assay was performed to calculate the amount of peptides. $10 \mu \mathrm{L}$ of peptides were removed from each sample and $0.1 \%$ FA added to equalise the volume and spiked with an internal standard protein (yeast alcohol dehydrogenase, ADH), with a known amount of 50 fmol injected for each run.

\section{Waters NanoAcquity UPLC and Synapt G2S}

Sample separation was performed using an Acquity UPLC Symmetry C18 trapping column $(180 \mu \mathrm{m} \times 20 \mathrm{~mm}, 5 \mu \mathrm{m})$ to remove salt and other impurities and a HSS T3 analytical column $(75 \mu \mathrm{m} \times 150 \mathrm{~mm}, 1.8 \mu \mathrm{m})$. Solvent A was compromised on $0.1 \%$ FA in HPLC grade water and solvent $\mathrm{B}$ contained $0.1 \% \mathrm{FA}$ in $\mathrm{ACN}$.

\begin{tabular}{|l|l|l|l|}
\hline $\begin{array}{l}\text { Time } \\
(\text { minute })\end{array}$ & $\begin{array}{l}\text { Flow rate } \\
(\mu \mathrm{L} / \text { minute })\end{array}$ & $\begin{array}{l}\text { Solvent A } \\
(\text { Water }+0.1 \% \text { FA })\end{array}$ & $\begin{array}{l}\text { Solvent B } \\
(\mathrm{ACN}+0.1 \% \mathrm{FA})\end{array}$ \\
\hline 3 & 0.3 & 97 & 3 \\
\hline 20 & 0.3 & 86 & 14 \\
\hline 30 & 0.3 & 80 & 20 \\
\hline 40 & 0.3 & 75 & 25 \\
\hline $51-52.2$ & 0.3 & 69 & 31 \\
\hline $53-53.1$ & 0.3 & 65 & 35 \\
\hline 54 & 0.3 & 63 & 37 \\
\hline 55 & 0.3 & 58 & 42 \\
\hline 63 & 0.3 & 31 & 69 \\
\hline
\end{tabular}




\begin{tabular}{|l|l|l|l|}
\hline 65 & 0.3 & 97 & 3 \\
\hline 80 & 0.3 & 50 & 50 \\
\hline 80.5 & 0.3 & 10 & 90 \\
\hline $82.2-87.5$ & 0.3 & 97 & 3 \\
\hline 99.5 & 0.3 & 50 & 50 \\
\hline 101.5 & 0.3 & 10 & 90 \\
\hline $103.5-110$ & 0.3 & 97 & 3 \\
\hline
\end{tabular}

Table above shows the gradient in 110 minutes of solvent A and B used in LC ESI-MS/MS analysis. The flow rate of solvents was $0.3 \mu \mathrm{L} /$ minute. Coupled directly to the Nano Acquity UPLC was a Water Synapt G2S mass spectrometer (Waters Corporation, Manchester, UK). The Synapt G2S includes a nano electrospray ionisation (ESI), StepWave ion guide, Quadrupole, TriWave and TOF (Supplementary Figure 2).

\section{Proteomic data processing and analysis}

Progenesis QI for Proteomics (Nonlinear Dynamics, Waters Corporation, UK) was employed to identify and quantify proteins. The human database from UniProtKB was downloaded and used in FASTA format. The proteomic raw data was searched using strict trypsin cleavage rules with a maximum of two missed cleavages. Cysteine (Carbamidomethyl C) was set as a fixed modification. Deamination N, Oxidation M and Phosphoryl STY were selected as variable modifications. Minimum of 2 fragments per peptide, minimum of 5 fragments per protein and minimum of 2 peptides per protein were set for parameters of identification. The maximum protein mass was set to $1000 \mathrm{kDa}$. The false rate discovery (FDR) for protein identification was set at a maximum rate of $1 \%$. Then, proteomic data generated from using the Progenesis QI was exported to Microsoft Excel for further data analysis.

For differential analysis, we used LIMMA (v.3.37.4) ${ }^{116}$. Because we compared obese and post surgery patients, we performed a paired analysis. We then applied a threshold of $0.1 \%$ on ordinary qvalue.

To define whole blood specific genes, we exported GTEx project ${ }^{117}$ expression table (in TPMs), converted it into SummarizedExperiment container using SummarizedExperiment R package ((v.1.11.6); Morgan M, Obenchain V, Hester J, Pagès H SummarizedExperiment: SummarizedExperiment container. (2019)) and used teGeneRetrieval function from the 
TissueEnrich R package (v.1.2.1) ${ }^{118}$. This package relies on Human Protein Atlas ${ }^{119}$ to grouped genes as follows: Tissue Enriched (Genes with an expression level greater than 1 TPM that also have at least five-fold higher expression levels in a particular tissue compared to all other tissues), Group Enriched (Genes with an expression level greater than 1 TPM that also have at least five-fold higher expression levels in a group of 2-7 tissues compared to all other tissues, and that are not considered Tissue Enriched) and tissue Enhanced (Genes with an expression level greater than 1 TPM that also have at least five-fold higher expression levels in a particular tissue compared to the average levels in all other tissues, and that are not considered Tissue Enriched or Group Enriched). With default parameters, we identified 693 whole blood specific genes. Finally we interesected genes coding for differentially abundant proteins and whole blood specific genes.

\section{Weighted correlation network analysis (WGCNA)}

WGCNA $^{47}$ is a correlation-based method that describes and visualizes networks of data points, whether they are gene expression estimates, metabolite concentrations or other phenotypic data. To increase statistical power, we merged the patient groups under the assumption that they share similar associations of metabolites and phenotypic traits. We followed the protocols of WGCNA to create metabolic networks. Metabolites are clustered into co-abundant "modules". Low correlations can be suppressed either in a continuous ("soft") manner or the discontinuous ("hard") thresholding used in constructing unweighted networks. To maintain scale-free topology, we estimated an applied power by computing soft-threshold with pickSoftThreshold function from WGCNA R package (v.1.64-1) ${ }^{120}$. To build network, we used blockwiseModules function with the following options: TOMType $=$ "signed", minModuleSize $=20$, reassignThreshold $=0$, mergeCutHeight $=0.25$ and corType="bicor". Each obtained module is notated by a unique color. Additionally, we assigned a name to each consensus module. Each module abundance profile can be summarised by one representative metabolite: the module eigen metabolite. Specifically, the module eigen metabolite was defined as the first right-singular vector of the standardized module expression data ${ }^{121}$. We performed 3 analysis: extreme phenotypes (obese individuals and lipodystrophy patients were combined to get minimal sample size for network analysis), donors (all BD individuals) and a consensus analysis. We identified 8, 22 and 16 modules with donors, patients and consensus data respectively. Regarding consensus analysis, we considered 988 metabolites, of these, 375 were assigned to 15 different modules and the remaining 613 were put in an ad hoc extra module because they did not show any correlation. 
We computed eigenmodule and biochemical parameters correlations (leptin-adiponectin ratio (LAR), glucose (GLC), triglycerides (TG), total cholesterol (TC), high density lipoprotein (HDL-C), low-density lipoprotein (LDL-C), alanine amino-transferase (ALT), aspartate amino-transferase (AST), Homeostatic Model Assessment for Insulin Resistance (HOMA-IR) and adipose tissue insulin resistance (AT-IR) indexes and high-sensitivity C-reactive Protein (hsCRP) and also weight (WGT), BMI and age) using cor function from stats $\mathrm{R}$ base package ( $\mathrm{R}$ version 3.5.0) and pearson method (default). P Value of each correlation was computed using corPvalueStudent function from WGCNA R package.

Pathways enrichment analysis were performed with MetaboAnalyst ${ }^{122}$ and in particular Pathway analysis module by submitting combined list HMDB identifiers for clusters $\mathrm{C} 1$ and $\mathrm{C} 2$, hyper-geometric test, relative-betweenness centrality topology analysis and KEGG database. In addition, we submitted these lists to the Reactome database.

\section{Multi-omic integration}

\section{Training datasets}

We identified $16 \mathrm{BD}$ individuals as controls, according to the following criteria: $\mathrm{BMI}<25$, glycaemia (GLUC) $<5.4 \mathrm{mmol} / \mathrm{L}$, TG $<1.7 \mathrm{mmol} / \mathrm{L}, \mathrm{LDL}<2.59 \mathrm{mmol} / \mathrm{L}, \mathrm{HDL}>1 \mathrm{mmol} / \mathrm{L}$ for men and $>1.3 \mathrm{mmol} / \mathrm{L}$ for women, HOMA score $<2.2$. For training the multi-omics predictive model (see below), we used a reduced training dataset comprising the subset of individuals having measurements across all omics layers. This reduced set comprised 6 controls, 6 obese individuals and 10 lipodystrophy patients. For the clinical data, we first used multiple imputation by chained equations, as implemented in the mice $\mathrm{R}$ package (with default options) to impute missing values before construction of the training dataset. We used the same method to impute missing clinical values in the NASH cohort.

\section{Variable selection: multivariable regression approach}

For each of the omics layers considered independently, we used elastic-net penalised logistic regression as implemented in the glmnet $\mathrm{R}$ package to identify putative signatures that discriminated between all patients (i.e. lipodystrophy + obese) versus controls. We adjusted for age and sex by including them as unpenalised covariates in the multivariable model. The elastic-net a parameter was fixed at $a=0.1$, while the $\lambda$ parameter was determined using cross-validation. Since different cross-validation splits resulted in different choices for $\lambda$, we performed multiple rounds of cross-validation, and used the value of $\lambda$ that resulted in the maximum number of selections. 


\section{Clinical predictive model}

We trained a ridge-penalised logistic regression model predictive of the binary response (i.e. patient/control status) using the clinical training dataset.

\section{Multi-omics predictive model}

We used the omic variables selected by the multivariable approach described above, together with the clinical covariates, to train a ridge-penalised logistic regression model predictive of the binary response (i.e. patient/control status). We fitted this model using the reduced training dataset. We used this model to make predictions for the 96 individuals for which we had measurements across all omics layers. To allow us to make predictions for those individuals for which we only had measurements on a subset of the omics datasets, we additionally fitted models to each combination of subsets.

\section{Validation of selected lipids}

To further investigate the lipidomic signature, we prioritised a reduced set of 9 lipid species that had been selected into the signature. These 9 species satisfied the following criteria: (1) they were selected into the lipidomic signature; and (2) using the Mann-Whitney test with Storey's q-value method to correct for multiple testing, we were able to reject the null hypothesis of no difference in distribution for these lipids in all of the following comparisons: (i) obese vs. control; (ii) lipodystrophy vs. control; and (iii) \{obese and lipodystrophy\} vs. control. All tests were performed using data from the present study only. Of these 9 species, we were able to match 8 with lipid species that had been quantified in a subset of 1,507 participants of the Fenland study ${ }^{82,84}$ which is a population-based cohort of 12,345 volunteers without diabetes born between 1950 and 1975 and recruited within the Cambridgeshire region between 2005 and 2015. We used linear regression analysis to test for association between plasma levels of the 8 lipid species selected into the lipidomic signature and all relevant CMS parameters quantified in both the reduced Fenland cohort, and the BD cohort, adjusting for age and sex, and using the Bonferroni method to control for multiple testing. To create a negative control set, we identified lipids that satisfied the following criteria: (1) they were not selected into the lipidomic signature; (2) they could be matched with lipid species that had been quantified in the reduced Fenland cohort; and (3) using the Mann-Whitney test with Storey's q-value method to correct for multiple testing, we were unable to reject the null hypothesis of no difference in distribution for these lipids in any of the following 
comparisons: (i) obese vs. control; (ii) lipodystrophy vs. control; and (iii) \{obese and lipodystrophy $\}$ vs. control. There were 37 lipid species that satisfied these criteria. We ranked these according to their mean absolute Pearson correlation with the 9 prioritised lipid species, and selected the 5 lowest ranking as our negative control set.

\section{Functional tests}

\section{Neutrophils Adhesion Method:}

Polymorphonuclear granulocytes were isolated via density gradient $(1.078 \mathrm{~g} / \mathrm{mL})$ from $3.2 \%$ sodium citrated whole blood within 2 hours of venipuncture. Neutrophil purity was assessed by haematology analyser (Sysmex, XN-450) to ensure purity levels were satisfactory ( $\geq 90 \%)$ for subsequent functional assays. Isolated cells were incubated in a water bath at $37 \mathrm{C}$ for 30 minutes with fluorescently labelled Calcein-AM (4ug/mL, Molecular probes). Cells were washed twice with 1x PBS and resuspended at 2x106/ml in HEPES complete medium supplemented with calcium (1mM). 1.6x105 fluorescently labelled neutrophils were then added to relevant duplicate wells in a 96-well plate containing the following stimuli; fMLP, $10 \mu \mathrm{M}$; DTT, 10mM; Pam3Cys, $20 \mu \mathrm{g} / \mathrm{ml}$; LBP+LPS, 50ng/mL and 20ng/mL; PAF, $1 \mu \mathrm{M}$; PMA, $1 \mu \mathrm{g} / \mathrm{mL}$; TNF, $10 \mathrm{ng} / \mathrm{mL}$ or HEPES only as a control in a final volume of in $100 \mu 1$. Cells were incubated for 30 minutes at $37 \mathrm{C}$ in a $5 \% \mathrm{CO} 2$ incubator, after which they were washed twice using $1 \mathrm{x}$ PBS before lysing in $100 \mu \mathrm{PBS}$ with $0.5 \%$ triton. A $100 \%$ adhesion control was generated by lysing $1.6 \times 105$ fluorescently labelled neutrophils in $0.5 \%$ triton. Fluorescent intensity was measured using a Tecan Infinite ${ }^{\circledR} 200$ PRO series plate reader (excitation of $485 / 20 \mathrm{~nm}$ and emission of $535 / 25 \mathrm{~nm}$ ). The mean of duplicate values were calculated and the $\%$ adhesion over the hepes control calculated using the following formula: $\%$ adhesion $=(($ RFU stimuli - RFU HEPES $) /$ RFU 100\% control $) \times 100$.

CD63 Expression:

50ul of whole blood was incubated with antibodies:

\begin{tabular}{|l|l|l|l|}
\hline CD16 & PE & VEP13 & Miltenyi \\
\hline CD63 & APC & H5C6 & Miltenyi \\
\hline CD11b & APC & ICRF44 & BD Pharmingen \\
\hline CD62L & FITC & Dreg 56 & BD Pharmingen \\
\hline CD32 & FITC & FLI8.26 & BD Pharmingen \\
\hline CD14 & APC & M $\varphi$ P9 & BD Pharmingen \\
\hline
\end{tabular}


for 20 minutes, followed by a red cell lysis (BD FACS lyse) and resuspension in $0.2 \%$ formyl saline. Samples were analysed using flow cytometry (Beckman Coulter, FC500) within 4 hours. Neutrophils were identified using scatter properties and CD16 positivity. BD CompBeads were used to generate compensation controls. The median fluorescence intensity (MFI) for each surface marker was calculated using Kaluza Analysis Software (Beckman Coulter).

\section{References}

1. Kelli, H. M. \& Kassas, I. Cardio Metabolic Syndrome: A Global Epidemic. J. Diabetes Metab. 6, (2016).

2. Go, A. S. et al. Heart disease and stroke statistics--2014 update: a report from the American Heart Association. Circulation 129, e28-e292 (2014).

3. Leal, J., Luengo-Fernández, R., Gray, A., Petersen, S. \& Rayner, M. Economic burden of cardiovascular diseases in the enlarged European Union. Eur. Heart J. 27, 1610-1619 (2006).

4. Grundy, S. M. et al. Diagnosis and management of the metabolic syndrome: an American Heart Association/National Heart, Lung, and Blood Institute Scientific Statement. Circulation 112, 2735-2752 (2005).

5. Azzu, V., Vacca, M., Virtue, S., Allison, M. \& Vidal-Puig, A. Adipose tissue-liver cross talk in the control of whole-body metabolism: implications in non-alcoholic fatty liver disease. Gastroenterology (2020) doi:10.1053/j.gastro.2019.12.054.

6. Alberti, K. G. M. M. et al. Harmonizing the metabolic syndrome: a joint interim statement of the International Diabetes Federation Task Force on Epidemiology and Prevention; National Heart, Lung, and Blood Institute; American Heart Association; World Heart Federation; International Atherosclerosis Society; and International Association for the Study of Obesity. Circulation 120, 1640-1645 (2009).

7. Hotamisligil, G. S. Inflammation and metabolic disorders. Nature 444, 860-867 (2006).

8. Stienstra, R. \& Stefan, N. Tipping the inflammatory balance: inflammasome activation distinguishes metabolically unhealthy from healthy obesity. Diabetologia 56, 2343-2346 (2013). 
9. Shimada, Y. J. et al. Bariatric surgery is associated with lower risk of acute care use for cardiovascular disease in obese adults. Cardiovasc. Res. 115, 800-806 (2019).

10. Pahan, K. Lipid-lowering drugs. Cell. Mol. Life Sci. 63, 1165-1178 (2006).

11. Majithia, A. \& Bhatt, D. L. Novel Antiplatelet Therapies for Atherothrombotic Diseases. Arterioscler. Thromb. Vasc. Biol. 39, 546-557 (2019).

12. Sanchez-Rangel, E. \& Inzucchi, S. E. Metformin: clinical use in type 2 diabetes. Diabetologia 60, 1586-1593 (2017).

13. Drucker, D. J. \& Nauck, M. A. The incretin system: glucagon-like peptide-1 receptor agonists and dipeptidyl peptidase-4 inhibitors in type 2 diabetes. Lancet 368, 1696-1705 (2006).

14. Kosmas, C. E. et al. Anti-inflammatory therapy for cardiovascular disease. Ann Transl Med 7, 147 (2019).

15. Stefan, N., Schick, F. \& Häring, H.-U. Causes, Characteristics, and Consequences of Metabolically Unhealthy Normal Weight in Humans. Cell Metab. 26, 292-300 (2017).

16. Kip, K. E. et al. Clinical importance of obesity versus the metabolic syndrome in cardiovascular risk in women: a report from the Women's Ischemia Syndrome Evaluation (WISE) study. Circulation 109, 706-713 (2004).

17. St-Pierre, A. C. et al. Insulin resistance syndrome, body mass index and the risk of ischemic heart disease. CMAJ 172, 1301-1305 (2005).

18. Katzmarzyk, P. T., Janssen, I., Ross, R., Church, T. S. \& Blair, S. N. The importance of waist circumference in the definition of metabolic syndrome: prospective analyses of mortality in men. Diabetes Care 29, 404-409 (2006).

19. Nichols, G. A. et al. Cardiometabolic Risk Factors Among 1.3 Million Adults With Overweight or Obesity, but Not Diabetes, in 10 Geographically Diverse Regions of the United States, 2012-2013. Prev. Chronic Dis. 14, E22 (2017).

20. Virtue, S. \& Vidal-Puig, A. Adipose tissue expandability, lipotoxicity and the Metabolic Syndrome--an allostatic perspective. Biochim. Biophys. Acta 1801, 338-349 (2010).

21. Choe, S. S., Huh, J. Y., Hwang, I. J., Kim, J. I. \& Kim, J. B. Adipose Tissue Remodeling: Its Role in Energy Metabolism and Metabolic Disorders. Front. Endocrinol. 7, 30 (2016). 
22. Hotamisligil, G. S., Shargill, N. S. \& Spiegelman, B. M. Adipose expression of tumor necrosis factor-alpha: direct role in obesity-linked insulin resistance. Science 259, 87-91 (1993).

23. Vishvanath, L. \& Gupta, R. K. Contribution of adipogenesis to healthy adipose tissue expansion in obesity. J. Clin. Invest. 129, 4022-4031 (2019).

24. Ramirez, G. A., Manfredi, A. A. \& Maugeri, N. Misunderstandings Between Platelets and Neutrophils Build in Chronic Inflammation. Front. Immunol. 10, 2491 (2019).

25. Puhr-Westerheide, D. et al. Neutrophils promote venular thrombosis by shaping the rheological environment for platelet aggregation. Sci. Rep. 9, 15932 (2019).

26. Bobryshev, Y. V., Ivanova, E. A., Chistiakov, D. A., Nikiforov, N. G. \& Orekhov, A. N. Macrophages and Their Role in Atherosclerosis: Pathophysiology and Transcriptome Analysis. Biomed Res. Int. 2016, 9582430 (2016).

27. Nording, H. M., Seizer, P. \& Langer, H. F. Platelets in inflammation and atherogenesis. Front. Immunol. 6, 98 (2015).

28. van Tuijl, J., Joosten, L. A. B., Netea, M. G., Bekkering, S. \& Riksen, N. P. Immunometabolism orchestrates training of innate immunity in atherosclerosis. Cardiovasc. Res. 115, 1416-1424 (2019).

29. Gros, A., Ollivier, V. \& Ho-Tin-Noé, B. Platelets in inflammation: regulation of leukocyte activities and vascular repair. Front. Immunol. 5, 678 (2014).

30. Koupenova, M., Clancy, L., Corkrey, H. A. \& Freedman, J. E. Circulating Platelets as Mediators of Immunity, Inflammation, and Thrombosis. Circ. Res. 122, 337-351 (2018).

31. Caielli, S., Banchereau, J. \& Pascual, V. Neutrophils come of age in chronic inflammation. Curr. Opin. Immunol. 24, 671-677 (2012).

32. Wright, H. L., Moots, R. J., Bucknall, R. C. \& Edwards, S. W. Neutrophil function in inflammation and inflammatory diseases. Rheumatology 49, 1618-1631 (2010).

33. Ghosh, S. et al. Gene expression profiling in whole blood identifies distinct biological pathways associated with obesity. BMC Med. Genomics 3, 56 (2010).

34. Matthews, D. R. et al. Homeostasis model assessment: insulin resistance and beta-cell function from fasting plasma glucose and insulin concentrations in man. Diabetologia $\mathbf{2 8}, 412-419$ 
(1985).

35. Søndergaard, E., Espinosa De Ycaza, A. E., Morgan-Bathke, M. \& Jensen, M. D. How to Measure Adipose Tissue Insulin Sensitivity. J. Clin. Endocrinol. Metab. 102, 1193-1199 (2017).

36. Bedogni, G. et al. The Fatty Liver Index: a simple and accurate predictor of hepatic steatosis in the general population. BMC Gastroenterol. 6, 33 (2006).

37. Sterling, R. K. et al. Development of a simple noninvasive index to predict significant fibrosis in patients with HIV/HCV coinfection. Hepatology 43, 1317-1325 (2006).

38. Artigao-Rodenas, L. M. et al. Framingham risk score for prediction of cardiovascular diseases: a population-based study from southern Europe. PLoS One 8, e73529 (2013).

39. Hippisley-Cox, J. et al. Predicting cardiovascular risk in England and Wales: prospective derivation and validation of QRISK2. BMJ 336, 1475-1482 (2008).

40. Elagizi, A. et al. An Overview and Update on Obesity and the Obesity Paradox in Cardiovascular Diseases. Prog. Cardiovasc. Dis. 61, 142-150 (2018).

41. Turro, E. et al. Whole-genome sequencing of patients with rare diseases in a national health system. Nature 583, 96-102 (2020).

42. Chen, L. et al. Genetic Drivers of Epigenetic and Transcriptional Variation in Human Immune Cells. Cell 167, 1398-1414.e24 (2016).

43. Cirulli, E. T. et al. Profound Perturbation of the Metabolome in Obesity Is Associated with Health Risk. Cell Metab. 29, 488-500.e2 (2019).

44. Moore, S. C. et al. Human metabolic correlates of body mass index. Metabolomics 10, 259-269 (2014).

45. Fiorenza, C. G., Chou, S. H. \& Mantzoros, C. S. Lipodystrophy: pathophysiology and advances in treatment. Nat. Rev. Endocrinol. 7, 137-150 (2011).

46. Huang-Doran, I., Sleigh, A., Rochford, J. J., O’Rahilly, S. \& Savage, D. B. Lipodystrophy: metabolic insights from a rare disorder. J. Endocrinol. 207, 245-255 (2010).

47. Zhang, B. \& Horvath, S. A general framework for weighted gene co-expression network analysis. Stat. Appl. Genet. Mol. Biol. 4, Article17 (2005).

48. Swystun, L. L. \& Liaw, P. C. The role of leukocytes in thrombosis. Blood 128, 753-762 (2016). 
49. Donkin, I. et al. Obesity and Bariatric Surgery Drive Epigenetic Variation of Spermatozoa in Humans. Cell Metab. 23, 369-378 (2016).

50. Campbell, L. E. et al. Identification of Novel Changes in Human Skeletal Muscle Proteome After Roux-en-Y Gastric Bypass Surgery. Diabetes 65, 2724-2731 (2016).

51. Kieffer-Kwon, K.-R. et al. Interactome maps of mouse gene regulatory domains reveal basic principles of transcriptional regulation. Cell 155, 1507-1520 (2013).

52. Quintin, J. et al. Candida albicans infection affords protection against reinfection via functional reprogramming of monocytes. Cell Host Microbe 12, 223-232 (2012).

53. Kvaløy, K., Page, C. M. \& Holmen, T. L. Epigenome-wide methylation differences in a group of lean and obese women - A HUNT Study. Sci. Rep. 8, 16330 (2018).

54. Busetto, L. et al. Practical Recommendations of the Obesity Management Task Force of the European Association for the Study of Obesity for the Post-Bariatric Surgery Medical Management. Obes. Facts 10, 597-632 (2017).

55. Adams, T. D. et al. Weight and Metabolic Outcomes 12 Years after Gastric Bypass. N. Engl. J. Med. 377, 1143-1155 (2017).

56. Raoux, L. et al. Effect of Bariatric Surgery-Induced Weight Loss on Platelet Count and Mean Platelet Volume: a 12-Month Follow-Up Study. Obes. Surg. 27, 387-393 (2017).

57. Periasamy, M. et al. Bariatric surgery decreases monocyte-platelet aggregates in blood: a pilot study. Obes. Surg. 24, 1410-1414 (2014).

58. Horie, T. et al. MicroRNA-33 regulates sterol regulatory element-binding protein 1 expression in mice. Nat. Commun. 4, 2883 (2013).

59. Singer, K. et al. Diet-induced obesity promotes myelopoiesis in hematopoietic stem cells. Mol Metab 3, 664-675 (2014).

60. Swanson, K. V., Deng, M. \& Ting, J. P.-Y. The NLRP3 inflammasome: molecular activation and regulation to therapeutics. Nat. Rev. Immunol. 19, 477-489 (2019).

61. Lee, W. L. \& Grinstein, S. Immunology. The tangled webs that neutrophils weave. Science vol. 303 1477-1478 (2004).

62. GTEx Consortium. Human genomics. The Genotype-Tissue Expression (GTEx) pilot analysis: 
multitissue gene regulation in humans. Science 348, 648-660 (2015).

63. Rendo-Urteaga, T. et al. Peripheral blood mononuclear cell gene expression profile in obese boys who followed a moderate energy-restricted diet: differences between high and low responders at baseline and after the intervention. Br. J. Nutr. 113, 331-342 (2015).

64. Das, S. K., Ma, L. \& Sharma, N. K. Adipose tissue gene expression and metabolic health of obese adults. Int. J. Obes. 39, 869-873 (2015).

65. Brown, A. J., Sepuru, K. M. \& Rajarathnam, K. Structural Basis of Native CXCL7 Monomer Binding to CXCR2 Receptor N-Domain and Glycosaminoglycan Heparin. Int. J. Mol. Sci. 18, (2017).

66. Kuan-Yu, I. et al. Activation of Adhesion GPCR EMR2/ADGRE2 Induces Macrophage

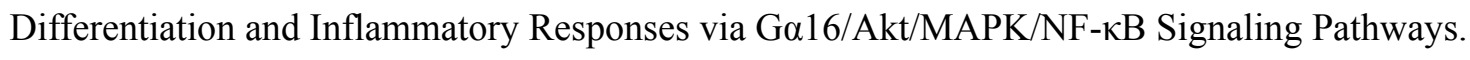
Front. Immunol. 8, 463 (2017).

67. Shah, D. et al. Obesity-induced adipokine imbalance impairs mouse pulmonary vascular endothelial function and primes the lung for injury. Sci. Rep. 5, 11362 (2015).

68. Wewer Albrechtsen, N. J. et al. Plasma Proteome Profiling Reveals Dynamics of Inflammatory and Lipid Homeostasis Markers after Roux-En-Y Gastric Bypass Surgery. Cell Syst 7, 601-612.e3 (2018).

69. Leentjens, J. et al. Trained Innate Immunity as a Novel Mechanism Linking Infection and the Development of Atherosclerosis. Circ. Res. 122, 664-669 (2018).

70. Bekkering, S. et al. Treatment with Statins Does Not Revert Trained Immunity in Patients with Familial Hypercholesterolemia. Cell Metab. 30, 1-2 (2019).

71. Brinkmann, V. Neutrophil Extracellular Traps Kill Bacteria. Science vol. 303 1532-1535 (2004).

72. Wang, H. et al. Obesity-induced Endothelial Dysfunction is Prevented by Neutrophil Extracellular Trap Inhibition. Sci. Rep. 8, 4881 (2018).

73. Cui, B.-B. et al. Neutrophil extracellular traps in sterile inflammation: the story after dying? Autoimmunity 45, 593-596 (2012).

74. Gavillet, M., Martinod, K., Renella, R., Wagner, D. D. \& Williams, D. A. A key role for Rac and Pak signaling in neutrophil extracellular traps (NETs) formation defines a new potential 
therapeutic target. Am. J. Hematol. 93, 269-276 (2018).

75. Gérard, A. et al. Detection of rare antigen-presenting cells through T cell-intrinsic meandering motility, mediated by Myo1g. Cell 158, 492-505 (2014).

76. Lood, C., Arve, S., Ledbetter, J. \& Elkon, K. B. TLR7/8 activation in neutrophils impairs immune complex phagocytosis through shedding of FcgRIIA. J. Exp. Med. 214, 2103-2119 (2017).

77. Liu, J., Liang, G., Siegmund, K. D. \& Lewinger, J. P. Data integration by multi-tuning parameter elastic net regression. BMC Bioinformatics 19, 369 (2018).

78. Wu, C. et al. A Selective Review of Multi-Level Omics Data Integration Using Variable Selection. High Throughput 8, (2019).

79. Zou, H. \& Hastie, T. Regularization and variable selection via the elastic net. Journal of the Royal Statistical Society: Series B (Statistical Methodology) vol. 67 301-320 (2005).

80. Waldmann, P., Mészáros, G., Gredler, B., Fuerst, C. \& Sölkner, J. Evaluation of the lasso and the elastic net in genome-wide association studies. Front. Genet. 4, 270 (2013).

81. Murphy, K. P. Machine Learning: A Probabilistic Perspective. (MIT Press, 2012).

82. Lindsay, T. et al. Descriptive epidemiology of physical activity energy expenditure in UK adults (The Fenland study). Int. J. Behav. Nutr. Phys. Act. 16, 126 (2019).

83. Hall, Z. et al. Lipid zonation and phospholipid remodeling in nonalcoholic fatty liver disease. Hepatology 65, 1165-1180 (2017).

84. Sanders, F. W. B. et al. Hepatic steatosis risk is partly driven by increased de novo lipogenesis following carbohydrate consumption. Genome Biol. 19, 79 (2018).

85. Bray, N. L., Pimentel, H., Melsted, P. \& Pachter, L. Near-optimal probabilistic RNA-seq quantification. Nat. Biotechnol. 34, 525-527 (2016).

86. Soneson, C., Love, M. I. \& Robinson, M. D. Differential analyses for RNA-seq: transcript-level estimates improve gene-level inferences. F1000Res. 4, 1521 (2015).

87. Zerbino, D. R. et al. Ensembl 2018. Nucleic Acids Res. 46, D754-D761 (2018).

88. Love, M. I., Huber, W. \& Anders, S. Moderated estimation of fold change and dispersion for RNA-seq data with DESeq2. Genome Biol. 15, 550 (2014). 
89. Leek, J. T., Johnson, W. E., Parker, H. S., Jaffe, A. E. \& Storey, J. D. The sva package for removing batch effects and other unwanted variation in high-throughput experiments.

Bioinformatics 28, 882-883 (2012).

90. Chen, E. Y. et al. Enrichr: interactive and collaborative HTML5 gene list enrichment analysis tool. BMC Bioinformatics 14, 128 (2013).

91. Kuleshov, M. V. et al. Enrichr: a comprehensive gene set enrichment analysis web server 2016 update. Nucleic Acids Res. 44, W90-7 (2016).

92. Li, H. \& Durbin, R. Fast and accurate long-read alignment with Burrows-Wheeler transform. Bioinformatics 26, 589-595 (2010).

93. Li, H. et al. The Sequence Alignment/Map format and SAMtools. Bioinformatics 25, 2078-2079 (2009).

94. Landt, S. G. et al. ChIP-seq guidelines and practices of the ENCODE and modENCODE consortia. Genome Res. 22, 1813-1831 (2012).

95. Kharchenko, P. V., Tolstorukov, M. Y. \& Park, P. J. Design and analysis of ChIP-seq experiments for DNA-binding proteins. Nat. Biotechnol. 26, 1351-1359 (2008).

96. Ramírez, F. et al. deepTools2: a next generation web server for deep-sequencing data analysis. Nucleic Acids Res. 44, W160-5 (2016).

97. Ross-Innes, C. S. et al. Differential oestrogen receptor binding is associated with clinical outcome in breast cancer. Nature 481, 389-393 (2012).

98. Heinz, S. et al. Simple combinations of lineage-determining transcription factors prime cis-regulatory elements required for macrophage and B cell identities. Mol. Cell 38, 576-589 (2010).

99. Müller, F. et al. RnBeads 2.0: comprehensive analysis of DNA methylation data. Genome Biol. 20, 55 (2019).

100. Triche, T. J., Jr, Weisenberger, D. J., Van Den Berg, D., Laird, P. W. \& Siegmund, K. D. Low-level processing of Illumina Infinium DNA Methylation BeadArrays. Nucleic Acids Res. 41, e90 (2013).

101. Maksimovic, J., Gordon, L. \& Oshlack, A. SWAN: Subset-quantile within array normalization 
for illumina infinium HumanMethylation450 BeadChips. Genome Biol. 13, R44 (2012).

102. Nordlund, J. et al. Genome-wide signatures of differential DNA methylation in pediatric acute lymphoblastic leukemia. Genome Biol. 14, r105 (2013).

103. Chen, Y.-A. et al. Discovery of cross-reactive probes and polymorphic CpGs in the Illumina Infinium HumanMethylation450 microarray. Epigenetics 8, 203-209 (2013).

104. Bolger, A. M., Lohse, M. \& Usadel, B. Trimmomatic: a flexible trimmer for Illumina sequence data. Bioinformatics 30, 2114-2120 (2014).

105. Xi, Y. \& Li, W. BSMAP: whole genome bisulfite sequence MAPping program. BMC Bioinformatics 10, 232 (2009).

106. Kent, W. J. et al. The human genome browser at UCSC. Genome Res. 12, 996-1006 (2002).

107. Du, P. et al. Comparison of Beta-value and M-value methods for quantifying methylation levels by microarray analysis. BMC Bioinformatics 11, 587 (2010).

108. Du, P., Kibbe, W. A. \& Lin, S. M. lumi: a pipeline for processing Illumina microarray. Bioinformatics 24, 1547-1548 (2008).

109. Akalin, A. et al. methylKit: a comprehensive R package for the analysis of genome-wide DNA methylation profiles. Genome Biol. 13, R87 (2012).

110. Wang, H.-Q., Tuominen, L. K. \& Tsai, C.-J. SLIM: a sliding linear model for estimating the proportion of true null hypotheses in datasets with dependence structures. Bioinformatics 27, 225-231 (2011).

111. O’Brien, K. A. et al. Metabolomic and lipidomic plasma profile changes in human participants ascending to Everest Base Camp. Sci. Rep. 9, 2297 (2019).

112. Eiden, M. et al. Mechanistic insights revealed by lipid profiling in monogenic insulin resistance syndromes. Genome Med. 7, 63 (2015).

113. Race, A. M., Styles, I. B. \& Bunch, J. Inclusive sharing of mass spectrometry imaging data requires a converter for all. J. Proteomics 75, 5111-5112 (2012).

114. Smith, C. A., Want, E. J., O’Maille, G., Abagyan, R. \& Siuzdak, G. XCMS: processing mass spectrometry data for metabolite profiling using nonlinear peak alignment, matching, and identification. Anal. Chem. 78, 779-787 (2006). 
115. Tautenhahn, R., Böttcher, C. \& Neumann, S. Highly sensitive feature detection for high resolution LC/MS. BMC Bioinformatics 9, 504 (2008).

116. Ritchie, M. E. et al. limma powers differential expression analyses for RNA-sequencing and microarray studies. Nucleic Acids Res. 43, e47 (2015).

117. Carithers, L. J. et al. A Novel Approach to High-Quality Postmortem Tissue Procurement: The GTEx Project. Biopreserv. Biobank. 13, 311-319 (2015).

118. Jain, A. \& Tuteja, G. TissueEnrich: Tissue-specific gene enrichment analysis. Bioinformatics 35, 1966-1967 (2019).

119. Uhlén, M. et al. Proteomics. Tissue-based map of the human proteome. Science 347, 1260419 (2015).

120. Langfelder, P. \& Horvath, S. WGCNA: an R package for weighted correlation network analysis. BMC Bioinformatics 9, 559 (2008).

121. Langfelder, P. \& Horvath, S. Eigengene networks for studying the relationships between co-expression modules. BMC Syst. Biol. 1, 54 (2007).

122. Chong, J., Yamamoto, M. \& Xia, J. MetaboAnalystR 2.0: From Raw Spectra to Biological Insights. Metabolites 9, (2019). 
bioRxiv preprint doi: https://doi.org/10.1101/2020.03.06.961805; this version posted March 4, 2021. The copyright holder for this preprint

$\mathrm{A}$ (which was not certified by peer review) is the author/funder. All rights reserved. No reuse allowed without permission.

C
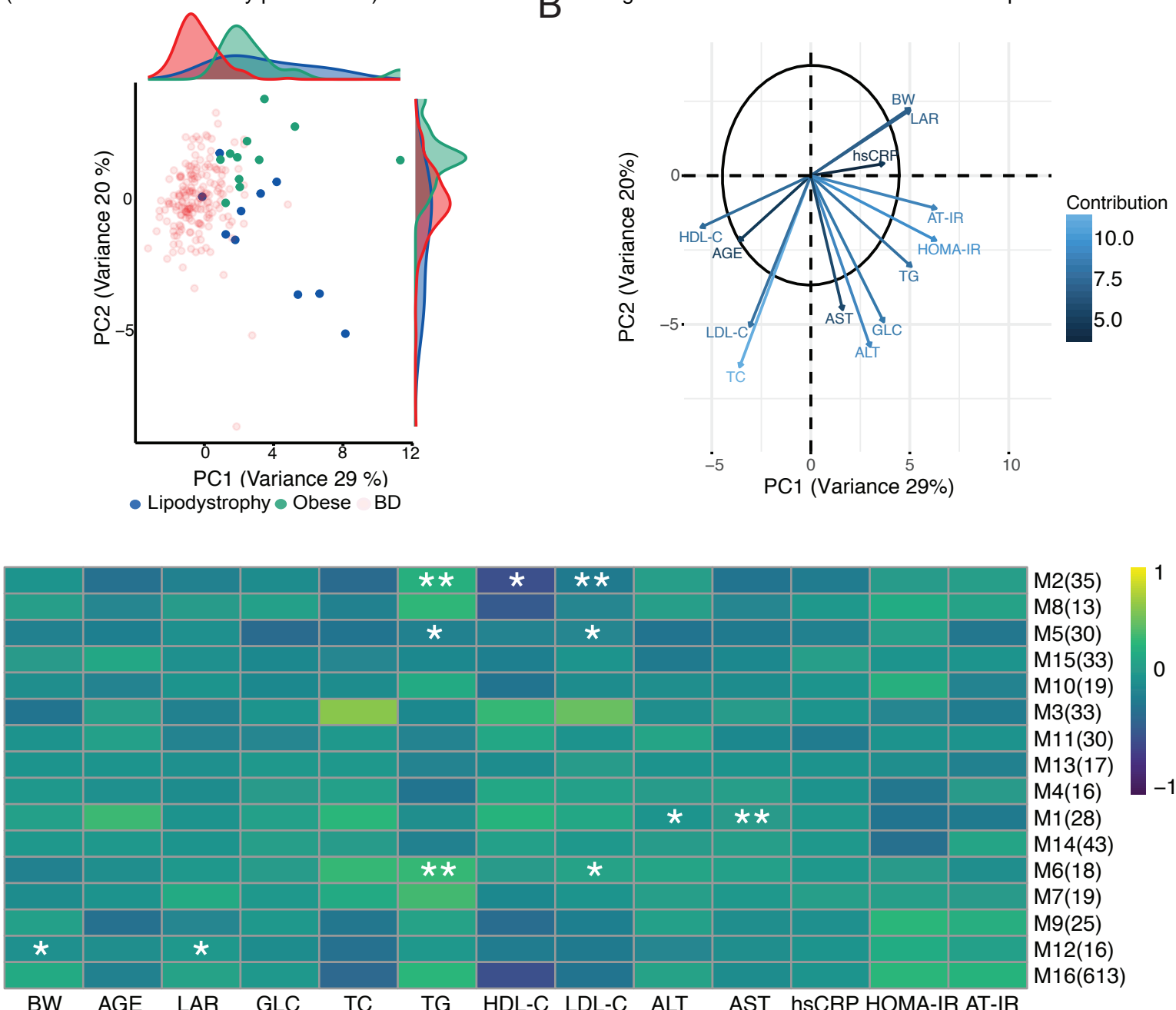

$\mathrm{D}$

E
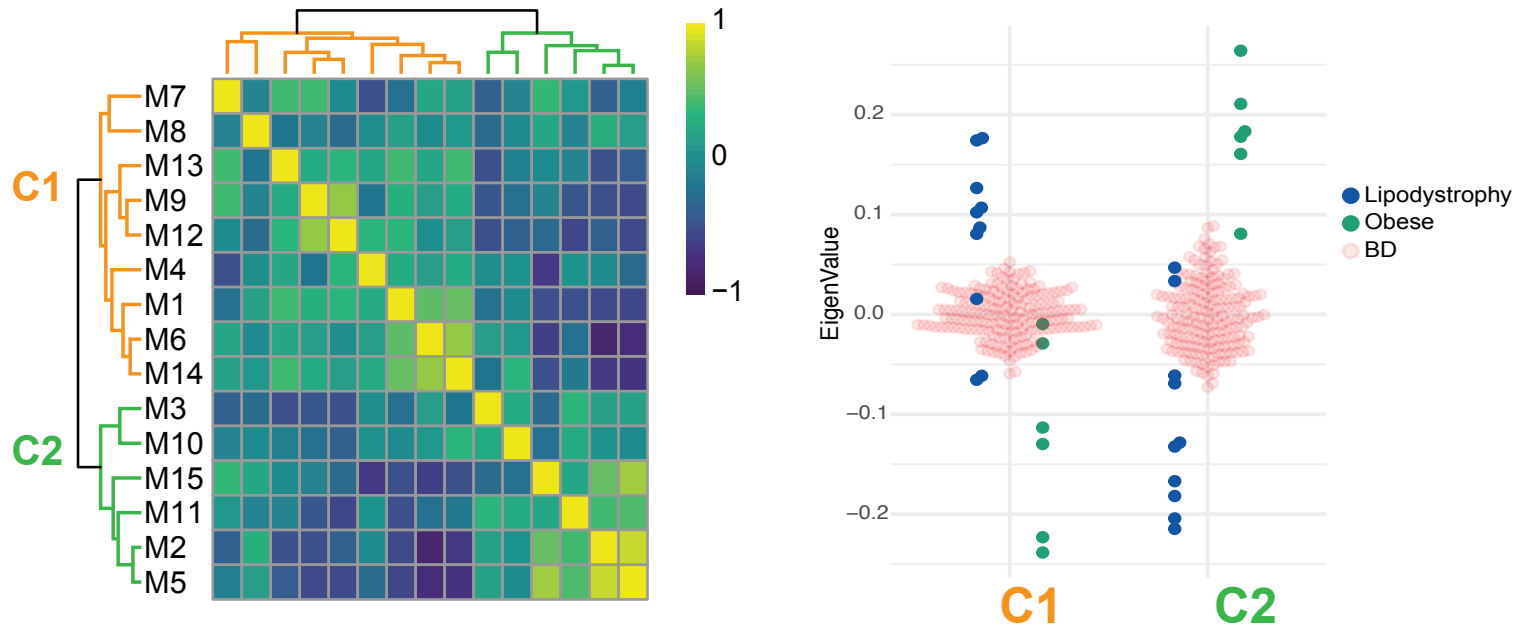

Figure 1 - Metabolic signatures in the obese and lipodystrophy groups.

A. Principal component analysis (PCA) of three groups: obese, green; lipodystrophy, blue; and blood donors (BD), light red. PCA was performed using the parameters below. B. Representation of PCA loadings on: age, weight (BW), body mass index (BMI), leptin-adiponectin ratio (LAR), glucose (GLC), triglycerides (TG), total cholesterol (TC), high density lipoprotein (HDL-C), low-density lipoprotein (LDL-C), alanine amino-transferase (ALT), aspartate amino-transferase (AST), Homeostatic Model Assessment for Insulin Resistance (HOMA-IR) and adipose tissue insulin resistance (AT-IR) indexes and high-sensitivity C-reactive Protein (hsCRP). Colour and arrow length scale represent contribution to variance on the first two principal components. C. Metabolite module-trait associations using WGCNA consensus analysis and 988 metabolites. Each row corresponds to a module eigen-metabolites (ME), and each column to a parameter. Number of metabolites in each module is indicated in brackets. Cell colour represents Pearson's correlation as shown by legend. Significance is annotated as follows: ${ }^{*} \mathrm{P} \leq 0.05,{ }^{* *} \mathrm{P} \leq 0.01,{ }^{* *} \mathrm{P} \leq 0.001,{ }^{* * *} \mathrm{P} \leq 0.0001$ (Fisher's test $\mathrm{p}$ value corrected for multi testing). D. Heatmap of extreme phenotype groups' MEs adjacencies in the consensus MEs network. The heatmap is color-coded by adjacency, yellow indicating high adjacency (positive correlation) and blue low adjacency (negative correlation). E. Beeswarm plot using average MEs per cluster presented in D. 

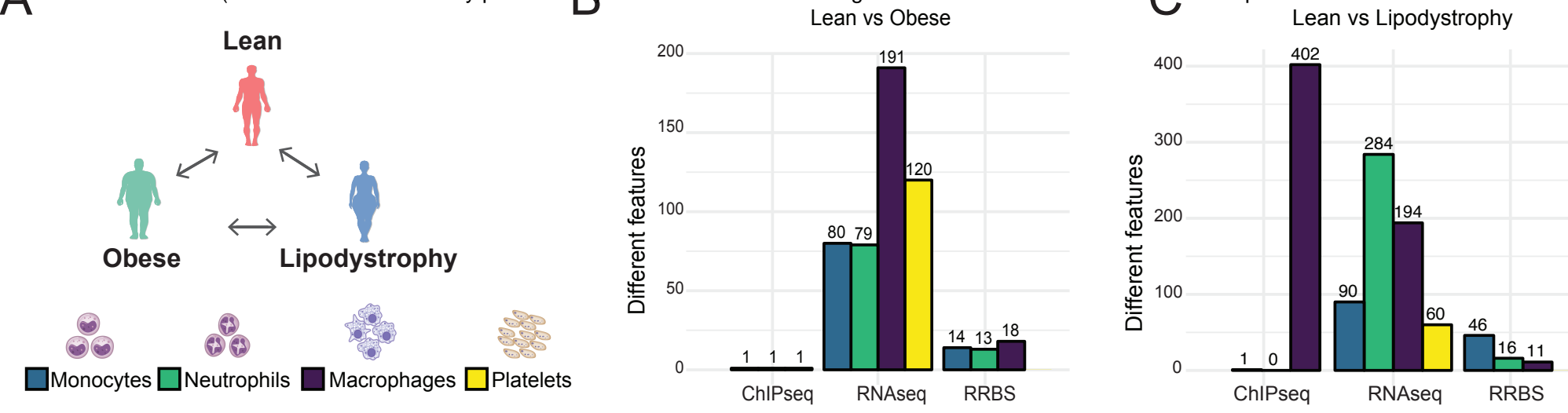

\begin{tabular}{|c|c|c|}
\hline 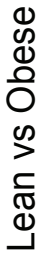 & $\begin{array}{r}\text { cholesterol biosynthetic process (GO:0006695) } \\
\text { Interferon alpha/beta signaling Homo sapiens R-HSA-909733 } \\
\text { Cholesterol Biosynthesis Pathway WP197 } \\
\text { Focal adhesion KEGG }\end{array}$ & $\begin{array}{r}49|8| 35 \\
142|9| 68 \\
142|3| 15 \\
142|8| 199\end{array}$ \\
\hline 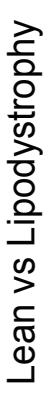 & $\begin{array}{r}\text { Interferon Signaling Homo sapiens R-HSA-913531 } \\
\text { regulation of viral genome replication (GO:0045069) } \\
\text { positive regulation of cytokine-mediated signaling pathway (GO:0001961) } \\
\text { Interferon alpha/beta signaling Homo sapiens R-HSA-909733 } \\
\text { regulation of nuclease activity (GO:0032069) } \\
\text { The human immune response to tuberculosis WP4197 } \\
\text { Cholesterol Biosynthesis Pathway WP197 } \\
\text { Major pathway of rRNA processing in the nucleolus Homo sapiens R-HSA-6791226 } \\
\text { Costimulation by the CD28 family Homo sapiens R-HSA-388841 } \\
\text { Glutathione metabolism KEGG } \\
\text { acetyl-CoA metabolic process (GO:0006084) } \\
\text { Interferon gamma signaling Homo sapiens R-HSA-877300 }\end{array}$ & $\begin{array}{l}160|5| 33 \\
51|3| 6 \\
51|3| 23 \\
5160|12| 63 \\
139|10| 68 \\
139|5| 72|10| 166|10| 166 \\
139|4| 56 \\
139|3| 14 \\
139|5| 93\end{array}$ \\
\hline
\end{tabular}

Figure 2 - Transcriptional and epigenetic signatures in extreme phenotype groups for three innate immune cell types and platelets.

A. Schematic overview of the comparisons made in the 4 different cell types (Monocytes: blue ; Neutrophils: green ; Macrophages: purple ; Platelets: yellow). B and C. Barplot showing the number of features significantly different: H3K27ac distribution (ChIP-Seq), gene expression (RNA-Seq) and DNA methylation (RRBS). Each bar is color coded to represent the different cell types as in A. B represents results when comparing lean and obese individuals. C represents results when comparing lean individuals and lipodystrophy patients. D. Functional GO term annotation of up-regulated genes when comparing lean versus obese group (top) and lean individuals versus lipodystrophy group (bottom), colour coded by cell types as above. The numbers near each dot indicate, from left to right: number of submitted genes, number of genes overlapping with the category and number of genes in the category. 
bioRxiv preprint doi: https://doi.org/10.1101/2020.03.06.961Bp5; this version posted March 4, 2021. The copyright holder for this preprint

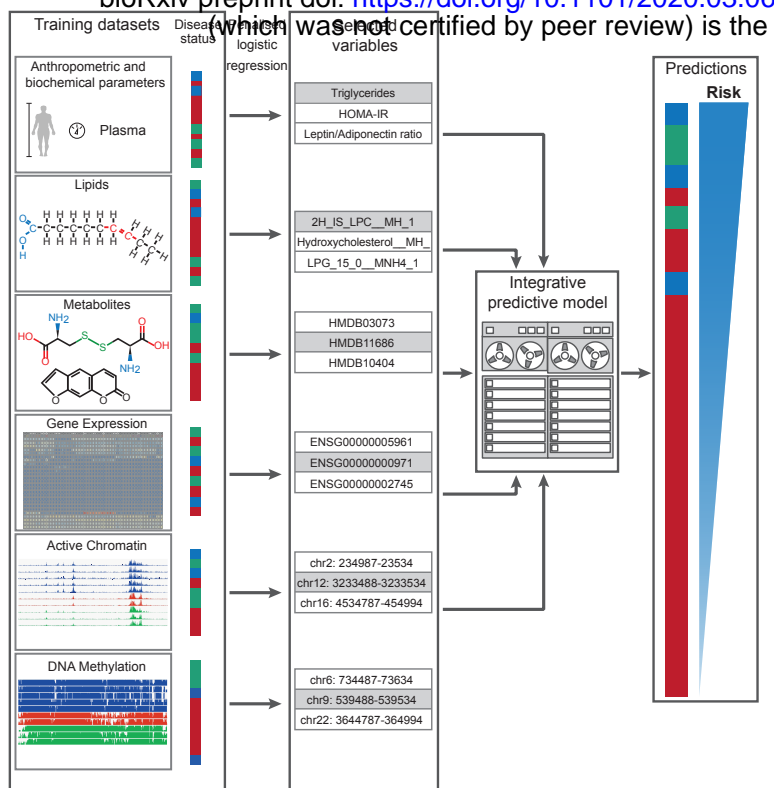

BD $\square$ Lipodystrophy Obese

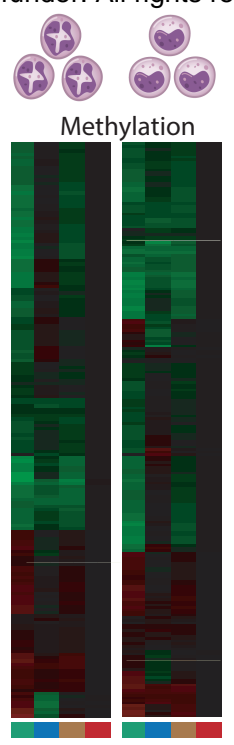

Obese

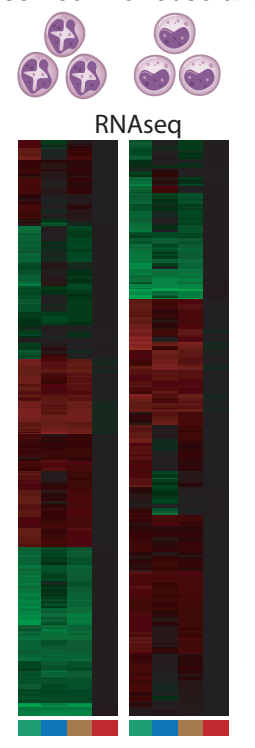

Lipodystrophy

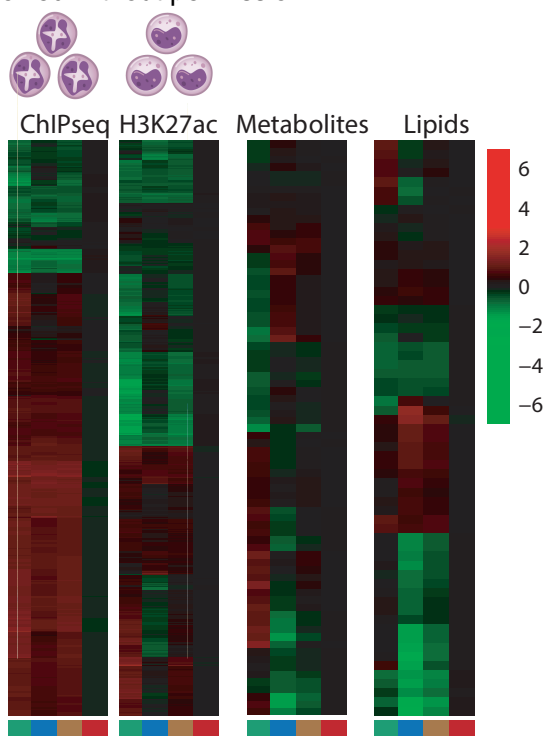

Extreme phenotypes

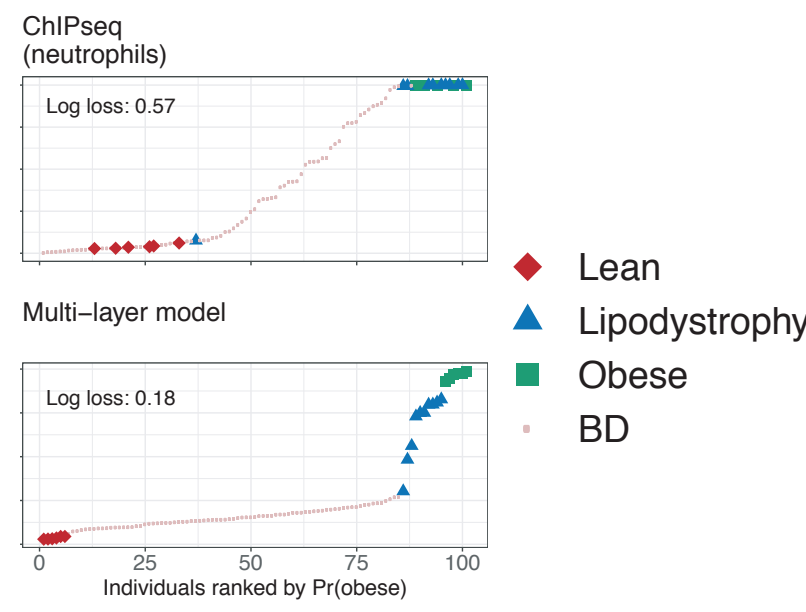

Prioritised lipids Fenland cohort BD cohort
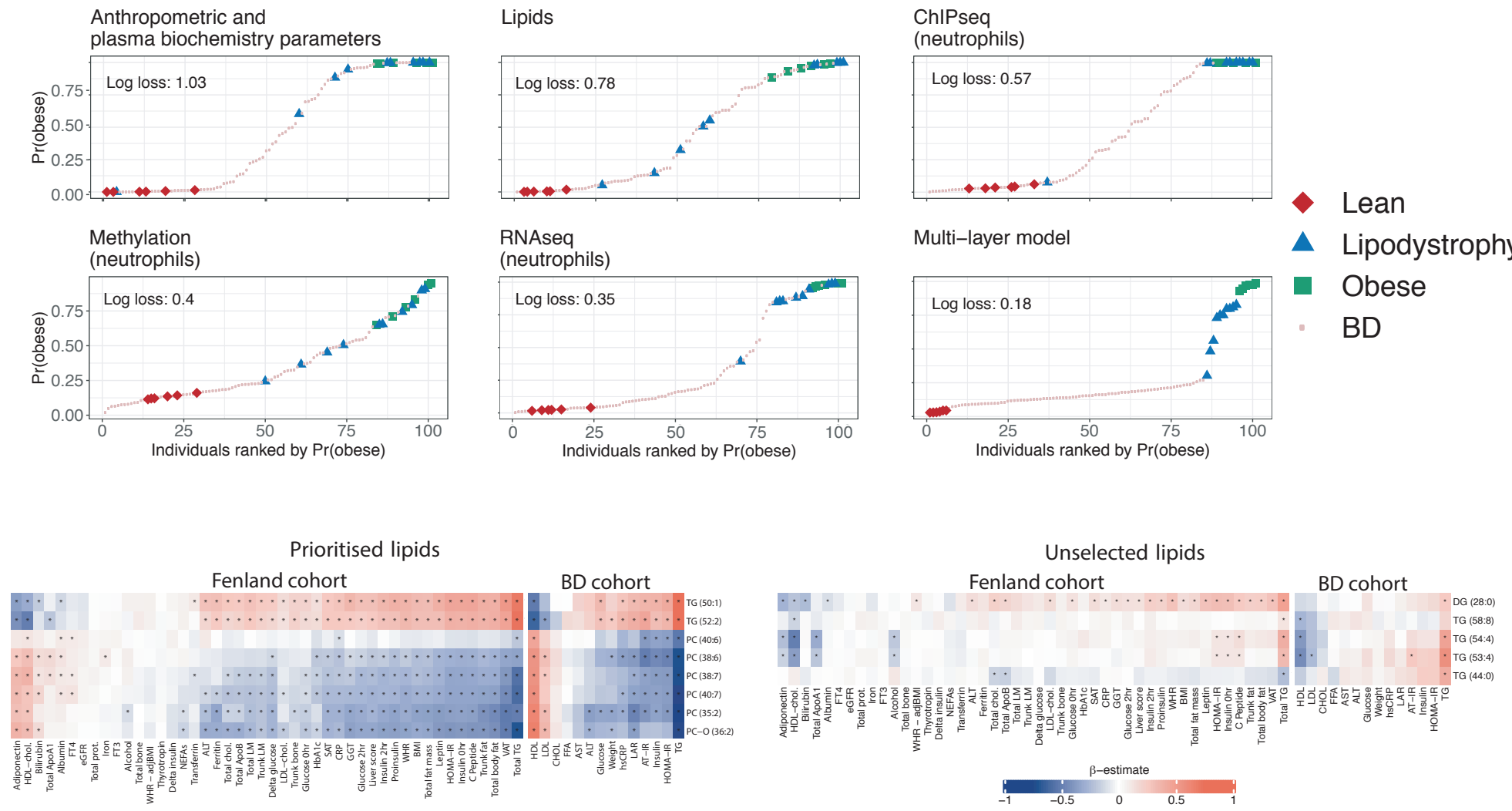

\section{Figure 4 - Multi-omic signature classification of extreme phenotypes.}

A. Presentation of the different layers used for multi-omic integration, the strategy leading to signature identification and schematic view of BD ranking. B. Heatmaps showing the mean of the Z-score distribution for each group, for all features selected in each layer. C. Plots showing individuals ranked by their predicted probability of belonging to the obese group, using models trained using data from individual layers, as well as a multi-layer predictive model (as indicated by the plot titles). Plots are ordered by decreasing log loss, with smaller values corresponding to better discrimination of individuals in the extreme phenotype group from all other individuals. D. Heatmap showing age and sex adjusted association values between (left) eigth prioritised lipid species and risk factors measured in the Fenland and present cohorts; and (right) a negative control set of five unselected lipid species and the same risk factors. Black asterisks indicate significant associations after correcting for multi- 
bioRxiv preprint doi: https://doi.org/10.1101/2020.03.06.961805; this version posted March 4, 2021. The copyright holder for this preprint

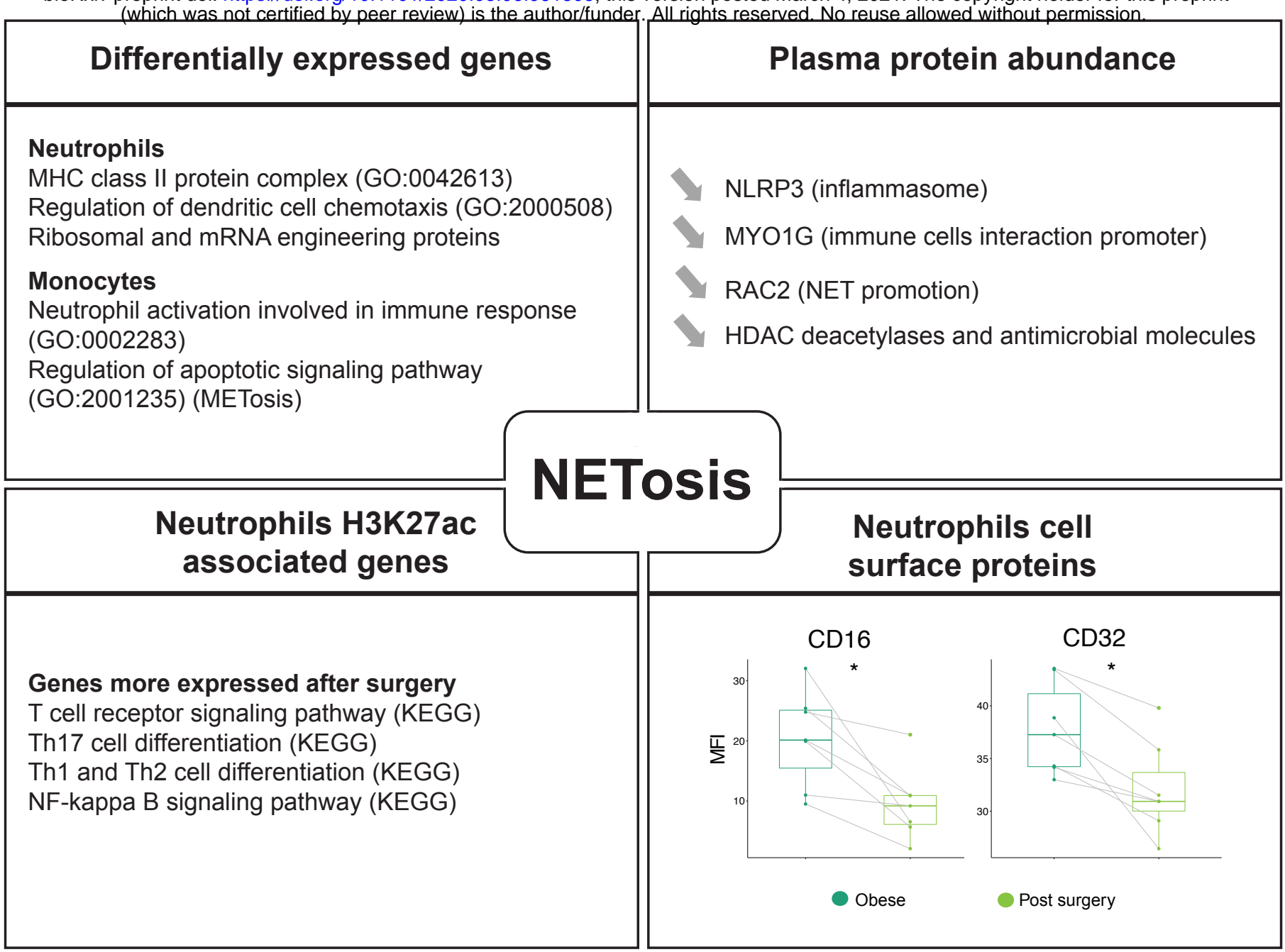

Figure 5 - Different omic layers contribution to NETosis reduction 6 months after bariatric surgery in mordibly obese individuals. Significance is annotated as follows: ${ }^{*} P \leq 0.05,{ }^{* *} P \leq 0.01,{ }^{* * *} P \leq$ $0.001,{ }^{* * * *} \mathrm{P} \leq 0.0001$. 